\title{
Article
}

\section{A small-molecule inhibitor of the BRCA2-RAD51 interaction modulates RAD51 assembly and potentiates DNA damage-induced cell death}

\author{
Duncan E. Scott, ${ }^{1,5,21}$ Nicola J. Francis-Newton, ${ }^{2,21}$ May E. Marsh, ${ }^{3,6}$ Anthony G. Coyne, ${ }^{1}$ Gerhard Fischer, ${ }^{3,7}$ \\ Tommaso Moschetti, ${ }^{3,8}$ Andrew R. Bayly, ${ }^{1,9}$ Timothy D. Sharpe, ${ }^{3,10}$ Kalina T. Haas, ${ }^{2,15}$ Lorraine Barber, ${ }^{2}$ \\ Chiara R. Valenzano, ${ }^{1,11}$ Rajavel Srinivasan, ${ }^{1,12,19}$ David J. Huggins, ${ }^{1,2,13}$ Miyoung Lee, ${ }^{2}$ Amy Emery, ${ }^{2}$ Bryn Hardwick, ${ }^{2}$ \\ Matthias Ehebauer, ${ }^{3,14}$ Claudio Dagostin, ${ }^{1,20}$ Alessandro Esposito, ${ }^{2}$ Luca Pellegrini, ${ }^{3}$ Trevor Perrior, ${ }^{4}$ \\ Grahame McKenzie, ${ }^{2,16}$ Tom L. Blundell, ${ }^{3}$ Marko Hyvönen, ${ }^{3, *}$ John Skidmore, ${ }^{1,5,23, *}$ Ashok R. Venkitaraman, ${ }^{2,17,18, *}$ \\ and Chris Abell ${ }^{1,22}$ \\ ${ }^{1}$ Yusuf Hamied Department of Chemistry, University of Cambridge, Lensfield Road, Cambridge CB2 1EW, UK \\ ${ }^{2}$ Medical Research Council Cancer Unit, University of Cambridge, Hutchison/MRC Research Centre, Hills Road, Cambridge CB2 0XZ, UK \\ ${ }^{3}$ Department of Biochemistry, University of Cambridge, 80 Tennis Court Road, Cambridge CB2 1GA, UK \\ ${ }^{4}$ Excellium Consulting, Brook Farm Barn, Lackford, Bury St Edmunds IP28 6HL, UK \\ ${ }^{5}$ Present address: The ALBORADA Drug Discovery Institute, University of Cambridge, Island Research Building, Hills Road, Cambridge CB2 \\ $\mathrm{OAH}, \mathrm{UK}$ \\ 6Present address: Paul Scherrer Institut, Villigen PSI, Switzerland \\ ${ }^{7}$ Present address: Boehringer Ingelheim RCV, Doktor-Boehringer-Gasse 5-11, 1121 Vienna, Austria \\ 8Present address: Illumina Cambridge Ltd, Illumina Center, Granta Park, Great Abington, Cambridge CB21 6DF, UK \\ 9Present address: Vertex Pharmaceuticals Limited, 86-88 Jubilee Avenue, Milton Park, Abingdon, Oxfordshire OX14 4RW, UK \\ ${ }^{10}$ Present address: Biophysics Facility, Biozentrum, University of Basel, Basel, Switzerland \\ ${ }^{11}$ Present address: Astex Pharmaceuticals, 436 Cambridge Science Park, Milton Road, Cambridge CB4 0QA, UK \\ ${ }^{12}$ Present address: School of Pharmaceutical Science and Technology, Health Science Platform, Tianjin University, 92 Weijin Road, Nankai \\ District, Tianjin 300072, People's Republic of China \\ ${ }^{13}$ Present address: Department of Physiology and Biophysics, Weill Cornell Medical College, New York, NY, USA \\ ${ }^{14}$ Present address: Ipsen Bioinnovation, Oxford, UK \\ ${ }^{15}$ Present address: Institut Jean-Pierre Bourgin, INRAE, AgroParisTech, Université Paris-Saclay, 78000 Versailles, France \\ ${ }^{16}$ Present address: PhoreMost Ltd., Babraham Research Campus, Cambridge CB22 3AT, UK \\ ${ }^{17}$ Present address: The Cancer Science Institute of Singapore, Center for Translational Medicine, National University of Singapore, Singapore \\ 117599, Singapore \\ ${ }^{18}$ Present address: Agency for Science, Technology and Research (A*STAR), 8A Biomedical Grove, Singapore 138648, Singapore \\ 19Present address: Singapore Eye Research Institute, The Academia, 20 College Road, Discovery Tower, Singapore 169856, Singapore \\ 20Present address: o2h group, Mill SciTech Park, Mill Lane Hauxton, Cambridge CB22 5HX, UK \\ ${ }^{21}$ These authors contributed equally \\ 22Prof Chris Abell died on October 26, 2020, during the revision period for this manuscript. The authors dedicate this paper to their good friend \\ and colleague. \\ ${ }^{23}$ Lead contact \\ ${ }^{*}$ Correspondence: mh256@cam.ac.uk (M.H.), js930@cam.ac.uk (J.S.), arv22@nus.edu.sg (A.R.V.) \\ https://doi.org/10.1016/j.chembiol.2021.02.006
}

\section{SUMMARY}

BRCA2 controls RAD51 recombinase during homologous DNA recombination (HDR) through eight evolutionarily conserved BRC repeats, which individually engage RAD51 via the motif Phe-x-x-Ala. Using structureguided molecular design, templated on a monomeric thermostable chimera between human RAD51 and archaeal RadA, we identify CAM833, a $529 \mathrm{Da}$ orthosteric inhibitor of RAD51:BRC with a $\mathrm{K}_{\mathrm{d}}$ of $366 \mathrm{nM}$. The quinoline of CAM833 occupies a hotspot, the Phe-binding pocket on RAD51 and the methyl of the substituted $\alpha$-methylbenzyl group occupies the Ala-binding pocket. In cells, CAM833 diminishes formation of damage-induced RAD51 nuclear foci; inhibits RAD51 molecular clustering, suppressing extended RAD51 filament assembly; potentiates cytotoxicity by ionizing radiation, augmenting $4 \mathrm{~N}$ cell-cycle arrest and apoptotic cell death and works with poly-ADP ribose polymerase (PARP)1 inhibitors to suppress growth in BRCA2-wildtype cells. Thus, chemical inhibition of the protein-protein interaction between BRCA2 and RAD51 disrupts HDR and potentiates DNA damage-induced cell death, with implications for cancer therapy. 


\section{INTRODUCTION}

The tumor suppressor protein, BRCA2, is essential for error-free repair of DNA double-stranded breaks (DSBs) by homologous DNA recombination (HDR) in human cells (Venkitaraman, 2014). BRCA2 acts during HDR to control the recombination enzyme, RAD51, a eukaryal protein evolutionarily conserved as RecA in eubacteria, and RADA in archaea (West, 2003). RAD51 executes the DNA strand exchange reactions that lead to HDR by assembling, in a sequential and highly regulated manner, as helical nucleoprotein filaments on single-stranded (ss) or double-stranded (ds) DNA substrates. The presynaptic RAD51 filament on ssDNA mediates strand invasion and homologous pairing with a duplex DNA template to execute strand exchange, the core biochemical event necessary for HDR.

Human BRCA2 contains two distinct regions that bind directly to RAD51. First, BRCA2 contains eight BRC repeats, evolutionarily conserved motifs of 26 residues each, whose sequence and spacing within a $\sim 1,100$-residue segment encoded by $B R C A 2$ exon 11 is conserved among mammalian orthologues (Bignell et al., 1997). Each of the eight human BRC repeats exhibits a varying affinity for RAD51 in vitro (Wong et al., 1997). Second, the carboxyl (C-) terminus of BRCA2 contains a RAD51-binding region spanning $\sim 90$ residues, which is distinct in sequence from the BRC repeats (Davies and Pellegrini, 2007; Esashi et al., 2007).

The interactions between BRCA2 and RAD51 control key steps essential for HDR. The BRC repeat-RAD51 interaction differentially regulates RAD51 assembly on DNA substrates in vitro, promoting assembly of the RAD51-ssDNA filament, while concurrently inhibiting the RAD51-dsDNA interaction (Carreira et al., 2009; Shivji et al., 2009). These opposing activities of the BRC repeats ensure that RAD51 assembly on its DNA substrates occurs in the correct order to promote strand exchange. Moreover, the C-terminal RAD51-binding region of BRCA2 stabilizes oligomeric assemblies of RAD51 in vitro in biochemical assays using purified proteins (Davies and Pellegrini, 2007; Esashi et al., 2007), and is required for the elongation of RAD51 filaments at cellular sites of DNA damage visualized by singlemolecule localization microscopy (Haas et al., 2018).

Of the eight BRC repeats in human BRCA2, BRC4 exhibits the highest affinity for RAD51 (Carreira and Kowalczykowski, 2011; Cole et al., 2017; Wong et al., 1997). The crystallographic structure of a complex between a BRC4 peptide and the core catalytic domain of RAD51 shows that the BRC4 sequence FHTA (human BRCA2 residues 1524-1527) engages with hydrophobic pockets on the RAD51 surface that accommodate the Phe and Ala residues (Pellegrini et al., 2002). An analogous FxxA motif in the RAD51 protein mediates oligomerization in the absence of DNA (Brouwer et al., 2018; Conway et al., 2004; Shin et al., 2003), and has recently been shown using electron cryo-microscopy to form the inter-subunit interface in functionally relevant DNA-bound assemblies of RAD51 (Short et al., 2016; Xu et al., 2017). In vitro, BRC4 peptides promote the strand selectivity of RAD51-DNA interactions at sub-stoichiometric concentrations relative to RAD51 (Carreira et al., 2009; Shivji et al., 2009). However, BRC4 peptides disrupt RAD51 oligomerization in vitro (Davies et al., 2001), and when overexpressed in cells, can inhibit the recruitment of RAD51 into DNA damage-induced foci by blocking the RAD51RAD51 interaction (Chen et al., 1999a).

The central importance of the BRC repeat-RAD51 interaction to HDR has prompted the identification of small-molecule and peptidic inhibitors that might have therapeutic value for cancer treatment. Most reported inhibitors target the interaction between RAD51 and DNA (Budke et al., 2012a, 2012b; Huang and Mazin, 2014; Huang et al., 2011; Ishida et al., 2009; Normand et al., 2014; Takaku et al., 2011). Recently described cell-penetrating antibodies also operate through a similar mechanism (Pastushok et al., 2019; Turchick et al., 2017, 2019). Inhibitors that suppress the D-loop activity of RAD51 have also been reported (Budke et al., 2019; Lv et al., 2016), although several optimized versions also exhibit DNA-intercalating activity (Budke et al., 2019). On the other hand, reports of small molecules and peptides have been published that claim to disrupt the interaction between RAD51 and the BRC repeats, or between RAD51 multimers (Bagnolini et al., 2020; Falchi et al., 2017; Nomme et al., 2010; Roberti et al., 2019; Trenner et al., 2018; Vydyam et al., 2019; Zhu et al., 2013, 2015; Ward et al., 2017). However, the lack of specific structural information concerning the interaction of these inhibitors with RAD51 has impeded the precise exploration of structure-activity relationships, and the efficient development of more potent compounds.

Here, we report the discovery, using a structure-led fragmentbased approach, of CAM833, a potent chemical inhibitor of the RAD51-BRC repeat interaction and RAD51 oligomerization. We show using $\mathrm{X}$-ray crystallography that CAM833 engages the Phe- and Ala-binding pockets on RAD51 to block its interaction with BRC repeats. We confirm that CAM833 potentiates cellular sensitivity to DNA damage induced by ionizing radiation, and suppresses the assembly of RAD51 into damage-induced filaments, as visualized by single-molecule localization microscopy. Our findings provide a well-characterized chemical tool compound to dissect biochemical events during HDR, and a potential lead for the development of new cancer therapeutics.

\section{RESULTS}

A monomeric thermostable chimera of human RAD51 and archaeal RADA recapitulates structural features of the human RAD51-BRC interaction

Structure-based approaches to identify modulators of the BRCA2-RAD51 interaction have been impeded by the lack of a monomeric unliganded form of HsRAD51. We have previously described the development of molecular surrogate systems for RAD51 based on an archaeal ortholog, RadA from Pyrococcus furiosus (Moschetti et al., 2016). In brief, we were able to produce the C-terminal ATPase domain of RadA as a stable monomeric protein, and by careful mutagenesis, to convert the surface of the protein to resemble human RAD51, with the ability to bind the BRCA2 BRC4 repeat with high affinity. Of note, we used the previously described constructs HumRadA2 for initial biophysics work and HumRadA22F for crystallography (Moschetti et al., 2016). In parallel, we also generated a chimeric RAD51 (ChimRAD51) that fuses the central part of the human RAD51 ATPase domain with two flanking sequences from archaeal RadA and used this in our primary screening assay and for biophysical screening. The binding of ChimRAD51 to the BRC4 peptide was characterized using a 
A
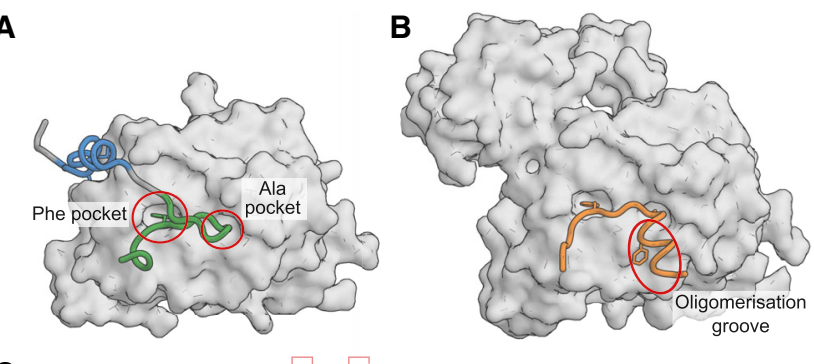

C

BRC4: CKEPTLLGFHTASGKKVKIAKESLDKVKNLFDEKEQ FXXA: PTLLGFHTASGKKV LFDE : $\quad$ AKESLDKVKNLFDEK OP : $\quad$ VPMGFTTATEFHQRRS

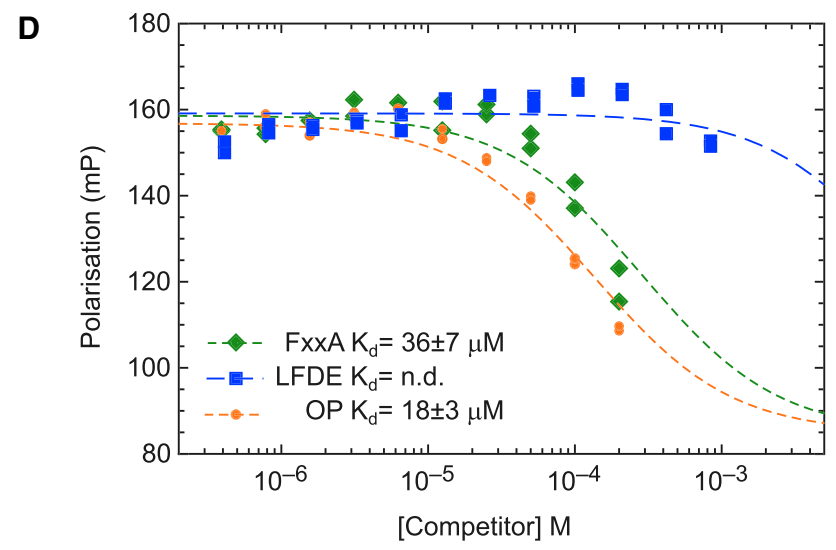

Figure 1. RAD51 interaction with BRC4

(A) Structure of RAD51 ATPase domain (surface) with BRC4 repeat of BRCA2 with FxxA binding motif colored green and the LFDE-motif in blue (PDB: 1nOw). (B) Structure of oligomeric RAD51 with oligomerization epitope (orange) of one protomer binding the next molecule in the filament (surface) (PDB: $5 \mathrm{nwl}$ ).

(C) Sequences of BRC4 repeat, and its FxxA and LFDE epitopes containing half peptides and the isolated RAD51 oligomerization peptide (OP).

(D) Competitive FP assay with labeled BRC4 repeat as probe which shows competitive binding to ChimRAD51 protein with the two BRC4 half-peptides (FxXA and LFDE, green and blue) and RAD51 oligomerization peptide (OP, orange). The dissociation constants measured for the FxxA half-peptide and for the oligomerization peptides were $36 \pm 7 \mu \mathrm{M}$ and $18 \pm 3 \mu \mathrm{M}$, respectively.

fluorescence polarization (FP) assay and by isothermal titration calorimetry (ITC), yielding comparable $K_{d}$ values of 4 and $6 \mathrm{nM}$, respectively validating the use of this protein for subsequent ligand affinity measurements (Moschetti et al., 2016). These surrogates provide robust platforms for structure-guided lead discovery via fragment screening, the biophysical characterization and validation of inhibitors, and for X-ray crystallography.

The three-dimensional structure of the C-terminal ATPase domain of RAD51 in complex with a BRC4 peptide has been determined by X-ray crystallography (Pellegrini et al., 2002). This structure shows that the BRC repeat binds in a bidentate fashion in which BRC4, via its FxxA motif, engages with a self-association site on RAD51, and then wraps around the protein to interact through a less-conserved LFDE motif with a second site on the RAD51 surface (Figure 1A). Cryo-EM structures of RAD51 filaments bound to DNA (Short et al., 2016; Xu et al., 2017) confirm that in self-association, the FxxA motif of one RAD51 interacts similarly with the two small "Phe" and "Ala" pockets on an adjacent protein unit, with the C-terminal segment of the oligomerization epitope binding to a hydrophobic groove in the opposite direction to that where the LFDE epitope of BRC4 binds. Earlier work has compared the relative affinities of the different human BRC repeats for RAD51 (e.g., Wong et al., 1997), and has demonstrated that both the FxxA and LFDE motifs in multiple BRC repeats contribute to both permissive and inhibitory interactions with RAD51 (Rajendra and Venkitaraman, 2010). In order to determine which of these two motifs might be most appropriate to develop inhibitors against, we measured the affinities of two peptides corresponding to $\mathrm{N}$ and $\mathrm{C}$-terminal epitopes of BRC4 using our FP assay. The $\mathrm{N}$-terminal "FxXA" half of the BRC4 repeat showed clear binding to RAD51 and competition of full-length BRC4 repeat with a $K_{d}$ of $36 \mu \mathrm{M}$. This compares favorably with our previous analysis of the affinities of tetra-peptides derived from the BRC4 FxxA epitope (which has the sequence FHTA), which bound to humanized RadA with 200 to $300 \mu \mathrm{M}$ affinity (Scott et al., 2016). The C-terminal half of BRC4 (LFDE peptide) showed very weak, if any, inhibition of BRC4 binding, at up to $1 \mathrm{mM}$ concentration (Figure $1 \mathrm{C}$ ), suggesting that the LFDE motif makes a minimal contribution on its own to this interaction, even though its mutation in the context of the entire BRC4 peptide can reduce RAD51 binding (Rajendra and Venkitaraman, 2010). We also tested the ability of RAD51 to bind its own oligomerization peptide (OP) epitope and determined a $\mathrm{K}_{\mathrm{d}}$ of $18 \mu \mathrm{M}$ for this interaction, demonstrating how additional binding energy can be derived from the interactions that the C-terminal part of the oligomerization peptide makes (Figure 1C).

The design and development of CAM833, a small molecule inhibitor of the interaction between BRCA2 and RAD51

Using the surrogate RAD51 systems described above and a combination of fragment-based drug discovery (Blundell et al., 2002; Coyne et al., 2010) and structure-guided drug design, we have optimized fragment hit molecules to generate high-affinity inhibitors of the RAD51-BRC-repeat interaction with a clearly defined orthosteric inhibition mechanism (Figure 2).

Initially, we carried out a biophysical fragment-screen against a previously described humanized version of PfRadA HumRadA2 (also known as MAYSAM [Moschetti et al., 2016; Scott et al., 2013]), leading to the discovery of a range of bicyclic aromatic and heteroaromatic fragment hits, binding exclusively into the Phe pocket at the FxxA site of RAD51 (Scott et al., 2013). Investigation of the structure-activity relationships (SAR) around these hits showed that naphthyl derivatives, particularly when substituted with polar groups, were able to bind to the Phe pocket with reasonable activity and ligand efficiency. For example, 2naphthol (2) bound to the HumRadA2 protein with a $\mathrm{K}_{d}$ of $460 \mu \mathrm{M}$ as measured by ITC (Scott et al., 2013), whereas 3amino-2-naphthoic acid (3) (Figure 2A) bound with a $\mathrm{K}_{\mathrm{d}}$ of $1.3 \mathrm{mM}$ (Figure S1). Crystallographic analysis of these fragments shows that the naphthyl rings bind in the same orientation as the aromatic side chain of phenylalanine in the FxxA motif of oligomerization peptide or BRC repeats (Figure 2B) (Scott et al., 2016).

In parallel, we explored the SAR of a series of $N$-acetylated tetrapeptides based on the FxxA epitope of BRC4 (Scott et al., 2016). This work established that the Ac-FHTA-NH $\mathrm{N}_{2}$ tetrapeptide 
A<smiles>CNC(Cc1ccccc1)C(=O)NC(Cc1c[nH]cn1)C(=O)NC(C(=O)N[C@@H](C)C(N)=O)C(C)O</smiles><smiles>Oc1ccc2ccccc2c1</smiles><smiles>C=CC(=O)O</smiles>

2

3<smiles>C=CCN1CNC(Cc2nc[nH]c2CC(NC(=O)c2cc3ccccc3cc2N)C(=O)NC(C)C(N)=O)C1=O</smiles>

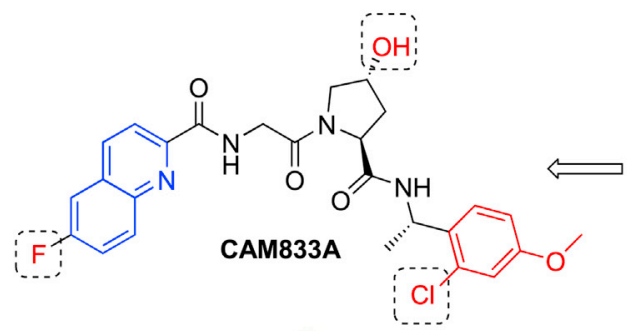<smiles>C=CC1(c2ccc([C@@H](C)NC(=O)[C@H]3CCCN3C(=O)CNC(=O)c3ccc4ccccc4n3)cc2)CCCO1</smiles><smiles>CC(NC(=O)C1CCCN1C(=O)C(C)(C)NC(=O)c1ccc2ccccc2c1)c1ccccc1</smiles>

B

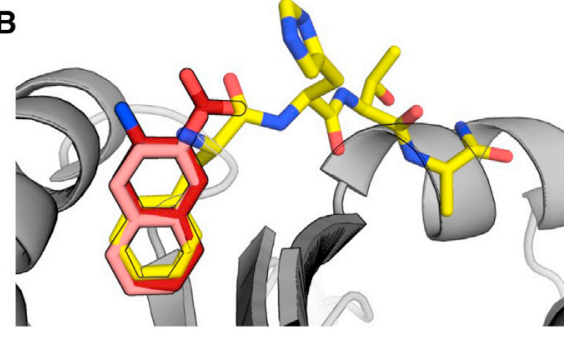

E

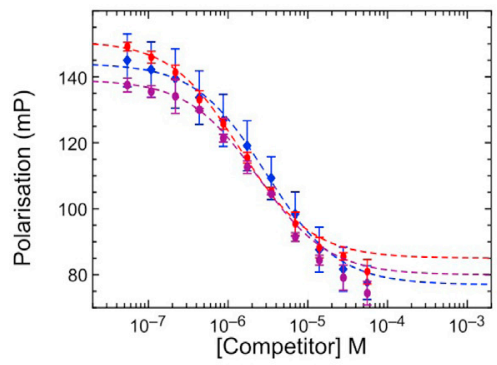

C

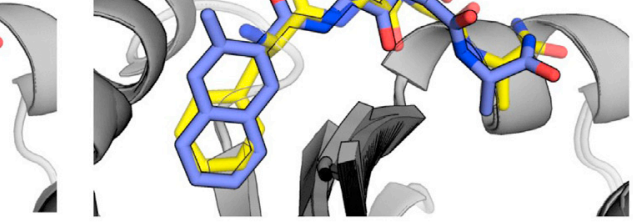

F Time (min)

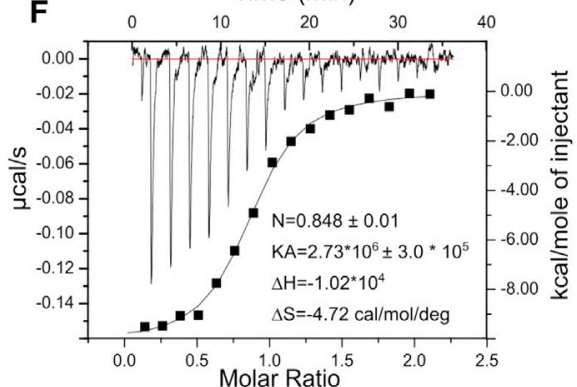

。

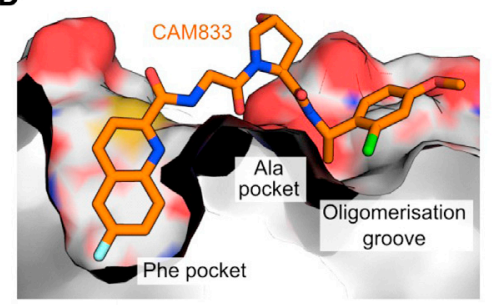

G

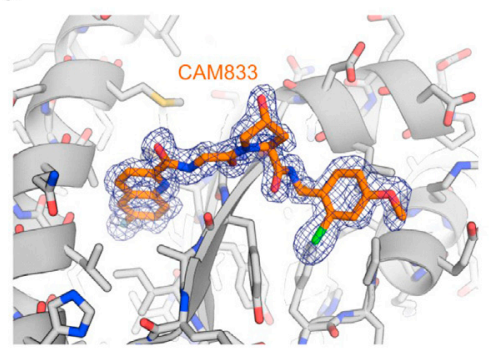

Figure 2. Development of CAM833

(A) Merging of 3-amino-2-naphthoic acid (3) with FHPA tetrapeptide to yield 4. Trimming of the naphthyl and histidine group and replacement of terminal amide with phenyl group yields $\mathbf{5}$. Increase of polarity by replacing naphthyl with quinoline and adding methoxy group the phenyl ring results in $\mathbf{6}$. Further optimization leads to CAM833.

(B) Overlaid crystal structures of HumRadA1 in complex with 2-naphthol (2, PDB: 4B32, pink), 3-amino-2-naphthoic acid (3, PDB: 6TV3, dark red) and FHTA tetrapeptide (1, PDB: 4B3B, yellow).

(C) Structure of 4 (PDB: 6TWR, deep purple) in complex with HumRadA1 overlaid with FHTA peptide (PDB: 4B3B, yellow).

(D) Structure of CAM833 (orange, PDB: 6TW9) in complex with HumRadA22F. Side view of CAM833 complex with HumRadA22F showing partially cut surface of the protein and interaction of the fluoroquinoline ring with the Phe-pocket and the chloro-phenyl group binding into the oligomerization groove.

(E) Competition of BRC4 peptide binding to ChimRAD51 using FP assay with CAM833. Three independent measurements (triplicate technical repeats) of the same binding are shown in three different colors.

(F) Isothermal titration calorimetric measurement of direct binding of CAM833 to ChimRAD51. The baseline corrected thermogram is shown above with $x$ and $y$ axes above and left of the graph. The solid squares depict integrated heats for each titration point and solid line the fit to single-site binding mode with corresponding $x$ and $y$ axes below and to left of the graph.

(G) Refined $2 F_{o} F_{c}$ electron density is shown for the ligand, contoured at $1 \sigma$.

(1) binds to HumRadA2 with a $\mathrm{K}_{\mathrm{d}}$ of $280 \mu \mathrm{M}$ as determined by ITC.

Based on an overlay of the X-ray crystal structures of $\mathbf{1}$ and the naphthyl fragments 2-naphthol (2) and 3-amino-2-naphthoic acid (3) (Figure 2B) we designed compound $\mathbf{4}$ in which the Phe of FHTA has been replaced by a rigid naphthyl-based amino acid, designed to more completely fill this pocket, and the threonine has been replaced by a proline in order to restrict the conformation of the peptide. The latter modification is known to provide a modest potency increase from the tetrapeptide structure activity relationship studies (Scott et al., 2016), with the benefit of removing two $\mathrm{H}$-bond donors from the structure, 
a change likely to be associated with an increase in cell permeability. Gratifyingly, the merged compound $\mathbf{4}$ was found to have improved $K_{d}$ of $3 \mu \mathrm{M}$ against HumRadA2 as determined by ITC (Figure S1), a considerable increase in potency compared with the native peptide. We determined an X-ray crystal structure of 4 bound to the HumRadA1 protein and this was found to interact in the predicted fashion, with a modest distortion of the peptide backbone in order to accommodate the more rigid left-hand side (orientation as in Figure 2) (Figure 2C).

Recognizing that the peptidic nature of $\mathbf{4}$ was likely to lead to poor pharmacokinetics and low permeability (clogP as calculated with ChemDraw $16-0.96$ and tPSA $172 \AA^{2}$ ), we sought to reduce the size and polarity of our compounds while introducing groups capable of forming additional interactions with the protein surface. This led to the design of compound $\mathbf{5}$, in which three polar elements judged unnecessary were removed: first, the His residue, which makes no key interactions in the tetrapeptide-protein crystal structure was cut back to an alanine; second, we removed the amino group from the terminal naphthoate unit. Finally, the terminal Ala amide was replaced with an $\alpha$-methylbenzylamino group that maintains the methyl group important for binding into the Ala pocket while replacing the terminal-amide with a lipophilic phenyl ring, inspired by the relatively non-polar nature of this region of the protein surface. Overall, compound 5 has only two intact amino acids and greatly reduced polarity (clogP $4.08, \mathrm{tPSA}=78.5 \AA^{2}$ ). Compound 5 has a $K_{d}$ of $220 \mathrm{nM}$ vs HumRadA2 by ITC (Figure S2), a 10-fold potency increase. By this stage, we had developed the more thoroughly humanized form of the protein ChimRAD51, which was subsequently used for our primary FP screening assays (Moschetti et al., 2016). We determined the $\mathrm{K}_{\mathrm{d}}$ of $\mathbf{5}$ against ChimRAD51 to be $1.9 \mu \mathrm{M}$ by ITC and $27 \mu \mathrm{M}(\mathrm{n}=20)$ as measured by FP. The reduced level of potency against this more humanized system was mirrored in data with the original 2-naphthol fragment (2), which we found to have a $\mathrm{K}_{\mathrm{d}}$ of $3.3 \mathrm{mM}$ for ChimRAD51 versus $460 \mu \mathrm{M}$ for HumRadA2.

Compound $\mathbf{5}$ was too insoluble in aqueous media to profile in cell-based assays. Accordingly, we made modifications designed to increase polarity, while avoiding the introduction of further hydrogen-bond donors likely to reduce permeability. We replaced the naphthyl ring with a quinoline, converted the Ala residue into a Gly, and introduced a 4-methoxy substituent on the right-hand phenyl ring, leading to 6 . Compound 6 has a clogP of 2.76 and an improved FP $K_{d}$ of $8.0 \mu \mathrm{M}(n=22)$ against ChimRAD51. Two independent X-ray structures of 6 demonstrated that this compound was still bound to the FxXA site with the quinoline accessing the Phe pocket in a similar orientation to the naphthyl in compound $\mathbf{4}$ albeit with a shifted binding mode discussed in more detail below (Figure S3).

More detailed exploration of the SAR around 6 led to the discovery of CAM833 with a 6-fluoro substituent on the quinoline and a 2-chloro group on the phenyl leading to a further increase in affinity. CAM833 has a $\mathrm{K}_{\mathrm{d}}$ against the ChimRAD51 protein of $355 \mathrm{nM}(\mathrm{n}=8)$ as measured by FP (Figure 2E) and $366 \mathrm{nM}$ by ITC (Figure 2F). The lipophilicity associated with these groups was balanced by the introduction of a trans-4-hydroxyl substituent on the proline ring serving to maintain solubility (clogP of CAM833 is 2.73 , and tPSA $120 \AA^{2}$ ).

As a biochemical test of CAM833, we evaluated its ability to disrupt full-length RAD51 oligomers. Using dynamic light scat- tering, we observe a shift of the average particle size from $\sim 40 \mathrm{~nm}$ for oligomeric RAD51 to $\sim 5 \mathrm{~nm}$ particles (corresponding closely to the size of a RAD51 monomer) in the presence of excess of CAM833 (Figure S4).

The X-ray crystal structures of 6 and CAM833 bound to HumRadA22F (the fully humanized RadA surrogate used for crystallography (Moschetti et al., 2016); Figures 2D, 2G, and S3) revealed an altered binding-mode compared to the lead compound 4 (Figure 2C). In this new binding mode, a shift of the backbone of CAM833 allows the NH of the right-hand benzyl amide to form a hydrogen bond to $\mathrm{Val}_{200_{189}}$ (subscript number refers to the equivalent human RAD51 residue, which differs from the surrogate protein residue numbering) via a bridging water-molecule rather than directly to the protein backbone (Figure S5). We attribute this to the truncation of the His residue back to a Gly, a change that can be tracked in the X-ray structures of intermediates from the optimization bound to HumRadA22F (data not shown).

Overall, examination of the structures reveals the source of the potency increases between the tetrapeptide 1 and CAM833. The phenyl ring of CAM833 sits flat on the protein surface with the ortho-chlorine atom sitting in a groove leading from this surface with both making beneficial hydrophobic interactions. The quinoline more completely fills the Phe pocket and the 6-fluoro substituent binds into a hydrophobic sub-pocket which has opened up due to minor movements in the residues lining the pocket (Figures $2 \mathrm{D}$ and $2 \mathrm{G}$ ). We determined selectivity and developability data for CAM833 in order to support its use as a validated chemical probe for the RAD51-BRCA-2 protein-protein interaction. Briefly, CAM833 is metabolically stable, does not significantly inhibit CYP450 enzymes, shows moderate solubility and permeability, and has no significant off-target interactions when screened at $10 \mu \mathrm{M}$ in the Cerep ExpresSPanel and has mouse pharmacokinetic data suitable for in vivo investigation (Table S1).

CAM833 causes a concentration-dependent decrease in RAD51 foci accompanied by increased DNA damage The assembly of RAD51 into microscopic foci at cellular sites of DNA damage is competitively inhibited by the overexpression of BRC repeat peptides (Chen et al., 1999a). Indeed, structural studies using X-ray crystallography (Brouwer et al., 2018; Pellegrini et al., 2002; Shin et al., 2003) as well as electron cryomicroscopy (Short et al., 2016; Xu et al., 2017) show that RAD51 assembly is mediated by protomer-protomer contacts that structurally mimic the RAD51-BRC repeat interaction. Because it interrupts these contacts in vitro, CAM833 is expected to suppress the function of RAD51 and prevent the formation of RAD51 foci in cells exposed to DNA damage.

We tested this prediction by monitoring RAD51 foci formation after the exposure of A549 non-small cell lung carcinoma (NSCLC) cells to ionizing radiation (IR), using a robust cell-based assay based on high-content microscopy with the Cellomics ArrayScan $\mathrm{V}^{\mathrm{Tl}}$, to objectively enumerate RAD51 foci (Jeyasekharan et al., 2013). IR-induced DNA breakage was monitored in the same experiment by enumerating foci containing $\gamma \mathrm{H} 2 \mathrm{AX}$, a phosphorylated form of histone $\mathrm{H} 2 \mathrm{AX}$ that is formed at DNA breaks (Rogakou et al., 1998).

Notably, CAM833 inhibited RAD51 foci formation $6 \mathrm{~h}$ after exposure to 3 Gy IR, in a concentration-dependent manner 
A
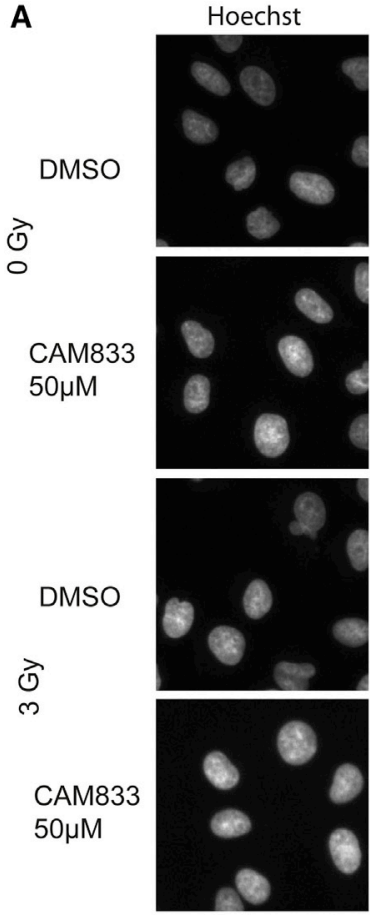

anti-RAD51
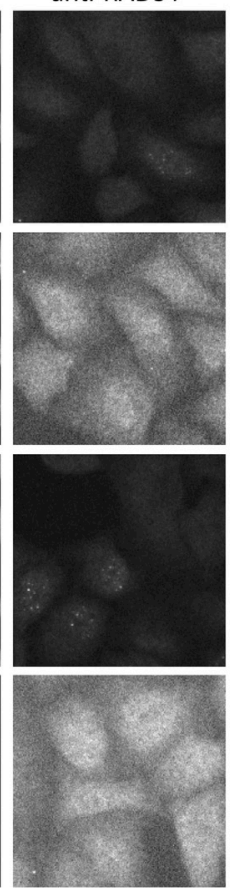
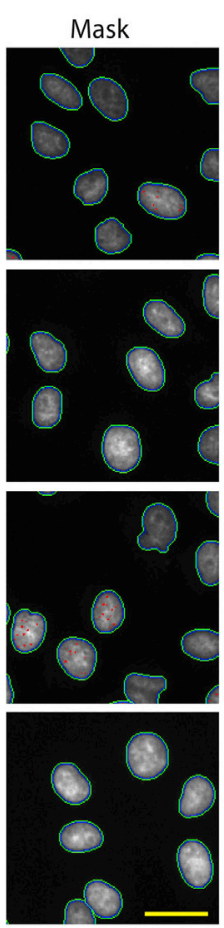

B

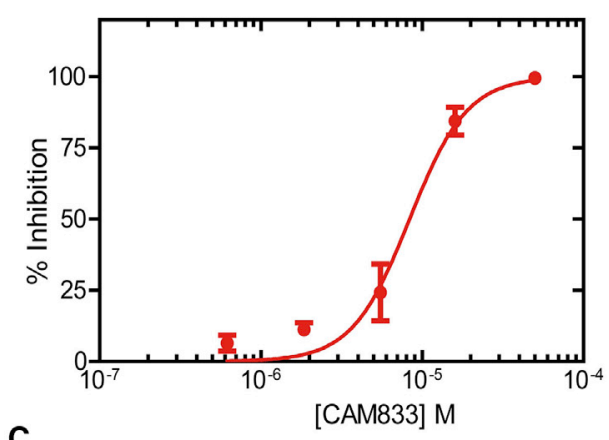

C

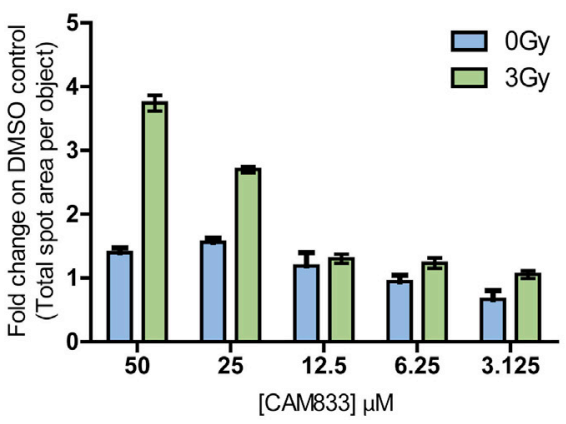

Figure 3. CAM833 causes a concentration-dependent decrease in RAD51 foci and subsequent increase in DNA damage

(A) Images from the Cellomics Arrayscan HCS microscope depicting A549 cells treated with CAM833 $(50 \mu \mathrm{M})$ or DMSO controls with or without ionizing radiation ( 3 Gy). Briefly, cells were co-stained with Hoechst-33342 to identify nuclei and anti-RAD51 antibody to detect RAD51 foci. The final column shows the Hoechststained cells with computationally identified nuclei outlined with green, and RAD51 foci with red, respectively. Scale bar, $20 \mu \mathrm{m}$ (estimated).

(B) An IC ${ }_{50}$ curve calculated from the images collected using the Cellomics HCS microscope as shown in (A). A549 cells were treated with CAM833, exposed to 3 Gy ionizing radiation (IR) and fixed after $6 \mathrm{~h}$ of incubation. CAM833 inhibits the formation of IR-induced RAD51 foci in A549 cells with an IC 50 of $6 \mu$ M. Percent inhibition on the $y$ axis was plotted against CAM833 concentration (as molar) on the $x$. Plots show mean \pm SEM.

(C) Cells treated by the same method were stained and counted for $\gamma-\mathrm{H} 2 \mathrm{AX}$ foci $24 \mathrm{~h}$ after exposure. Each pair of bars corresponds to cells exposed to one of five different concentrations (lowest, $3.125 \mu \mathrm{M}$ on the right, to highest, $50 \mu \mathrm{M}$, on the left) of CAM833 alone (0 Gy), or CAM833 plus 3 Gy IR (3 Gy). Bars depict the mean values of the fold change in $\gamma-\mathrm{H} 2 \mathrm{AX}$ foci number over control cells treated with DMSO alone, \pm SEM. CAM833 causes a concentration-dependent increase in unresolved DNA damage after $24 \mathrm{~h}$. Results are representative of three independent repeats.

with an $\mathrm{IC}_{50}$ of $6 \mu \mathrm{M}$ (Figures $3 \mathrm{~A}$ and $3 \mathrm{~B}$, plotted as mean $\pm \mathrm{SEM}$, $\mathrm{n}=27$ ). No RAD51 foci were detected at $\sim 50 \mu \mathrm{M}$ CAM833, corresponding to a maximal level of inhibition. Furthermore, $50 \mu \mathrm{M}$ CAM833 increased $\gamma \mathrm{H} 2 \mathrm{AX}$ foci formation $24 \mathrm{~h}$ after exposure by approximately 4-fold compared with control-treated cells (Figure 3C), suggestive of the persistence of unrepaired DNA damage. These findings are consistent with prior results in cells overexpressing BRC peptides (Chen et al., 1999b), and provide evidence that CAM833 engages its target in the cellular milieu to suppress RAD51 assembly and inhibit DNA repair by HDR.

\section{CAM833 inhibits RAD51 molecular clustering after DNA damage}

We have recently visualized the assembly of RAD51 molecules on DNA substrates at cellular sites of DNA damage using single-molecule localization microscopy (SMLM) by direct stochastic optical reconstruction (d-STORM) (Haas et al., 2018). Clusters of approximately 5 to 10 RAD51 molecules are first recruited to DNA damage sites 0.5 to $1 \mathrm{~h}$ after damage induction, which progressively extend into filaments $>200 \mathrm{~nm}$ in length 3 to $5 \mathrm{~h}$ afterwards. SMLM shows that RAD51 clustering is suppressed by the overexpression of $B R C$ repeat peptides, indicative of its depen- dence on protomer-protomer contacts that structurally mimic the RAD51-BRC repeat interaction inhibited in vitro by CAM833.

Therefore, to test the effect of CAM833 on RAD51 clustering, we used SMLM on patient-derived EUFA423 cells (Figure 4A) bearing compound heterozygosity for the cancer-associated BRCA2 truncating alleles 7691insAT and 9000insA (Haas et al., 2018; Howlett et al., 2002). We developed, as isogenic controls, EUFA423 cells complemented by the expression of full-length BRCA2 (EUFA423 + BRCA2) (Hattori et al., 2011). We enumerated the number of RAD51 molecules detected by SMLM in clusters induced by the exposure of EUFA423 cells or EUFA423 + BRCA2 controls (Figure 4A) to $3 \mathrm{~Gy} I \mathrm{R}$, in the presence or absence of $25 \mu \mathrm{M}$ CAM833, using a suite of bespoke image analysis algorithms that we have recently reported (Haas et al., 2018). As expected, the accumulation of RAD51 molecules in damage-induced clusters is significantly reduced in BRCA2deficient EUFA423 cells compared with EUFA423 + BRCA2 controls (Figure 4B) (Hattori et al., 2011). Notably, addition of $25 \mu \mathrm{M}$ CAM833 significantly reduces RAD51 accumulation in damageinduced foci to a further extent in both cell types, providing additional evidence that CAM833 inhibits RAD51 protomer-protomer contacts during filament assembly. 
A

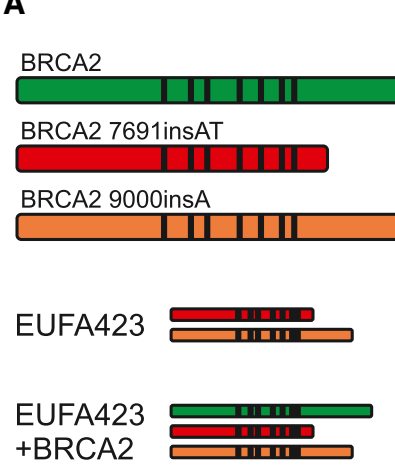

B

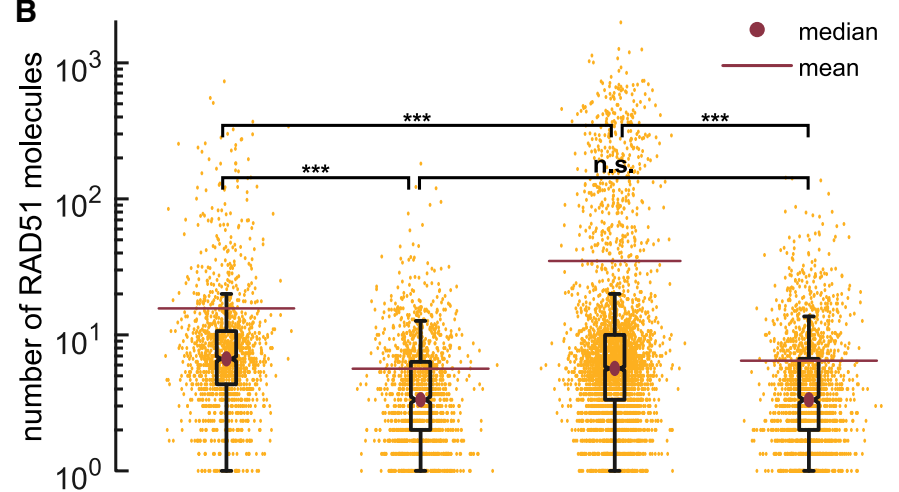

EUFA423

CAM $\overline{833(25 \mu \mathrm{M})}$

EUFA423+BRCA2

\section{C}
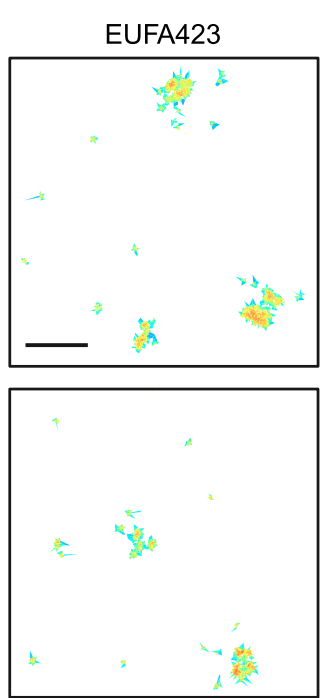

EUFA423+CAM833

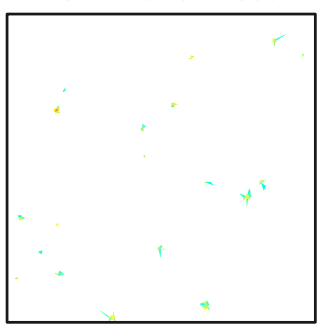

EUFA423+BRCA2

EUFA423+BRCA2+CAM833
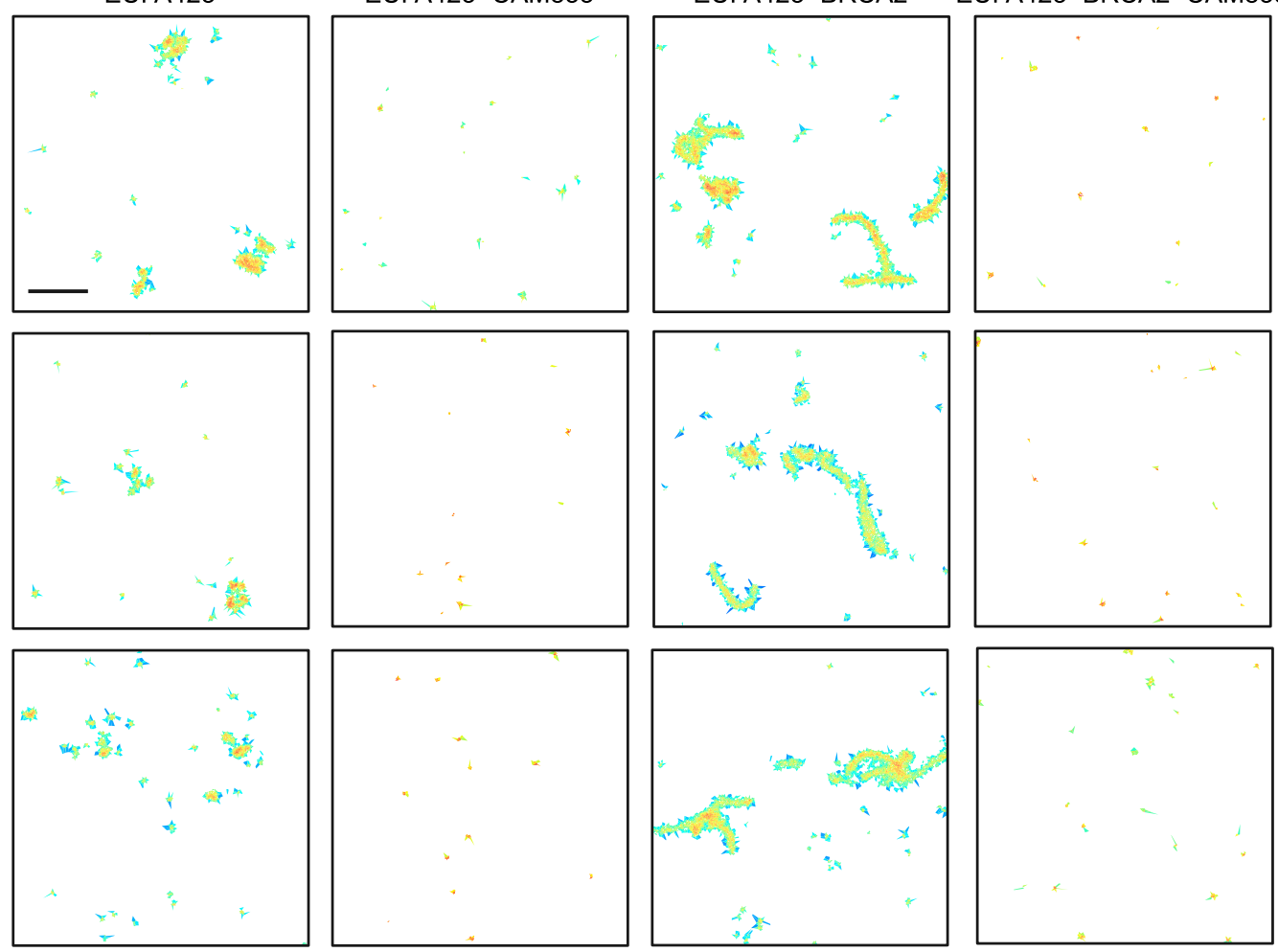

normalized RAD51 density

D


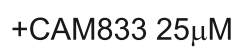

Figure 4. CAM833 inhibits RAD51 molecular clustering at DNA damage sites visualized by SMLM

(A) Diagrammatic representation of the biallelic truncating mutations (red and orange) affecting BRCA2 in the patient-derived cell line EUFA423, and their functional complementation by full-length BRCA2 (green) in EUFA423 + BRCA2 cells. Black vertical lines depict the approximate positions of the BRC repeats. 
The inhibitory effects of CAM833 are clearly observed by visualization of damage-induced RAD51 clusters as two-dimensional Voronoi polygons scaled to the maximum molecular density (Figure 4C). The compound effectively suppresses RAD51 clustering in both cell types, and in particular, prevents the formation of elongated filaments in control EUFA423 + BRCA2 cells. Example dSTORM pixel images (Figure 4D) further illustrate these effects, providing multiple lines of evidence for CAM833 target engagement and mechanism of action in cells.

\section{CAM833 inhibits DNA repair by homologous} recombination

The inhibition of RAD51 clustering at DNA damage sites by CAM833 prompted us to examine its effect on DNA repair by homologous recombination using the mClover-Lamin A assay (Arnoult et al., 2017; Buisson et al., 2017). In this assay, accurate repair of a nuclease-induced break in a recombination substrate through homologous recombination but not other mechanisms, allows reconstitution of an mClover-Lamin A fluorescent fusion protein, which localizes to the nuclear membrane (Figure 5A). The efficiency of homology-directed repair is readily measured by the enumeration of cells expressing the fusion protein (Figure $5 \mathrm{~B}$ ). We observe (Figure $5 \mathrm{C}$ ) that CAM833 induces a dosedependent decrease in the percentage of cells expressing the fluorescent fusion protein marker. Expression declines in a statistically significant manner at CAM833 doses between 6.25 and $12.5 \mu \mathrm{M}$, to a maximal inhibition at doses above $25 \mu \mathrm{M}$. Taken together with its effects on RAD51 clustering (Figure 4), the findings in Figure 5 provide evidence that CAM833 engages its intracellular target to inhibit DNA repair by homologous recombination.

CAM833 potentiates radiation-induced cell-cycle arrest and increases apoptosis over time

Genetic inactivation of RAD51 enhances cellular sensitivity to ionizing radiation, accompanied by cell-cycle arrest at the G2 checkpoint for DNA damage (Sonoda et al., 1998; Su et al., 2008). We hypothesized that similar effects would be triggered by the exposure of cells to CAM833. Indeed, when HCT116 coIon carcinoma cells exposed to $20 \mu \mathrm{M}$ CAM833 and 3 Gy IR were cell-cycle profiled by flow cytometry 4 to $72 \mathrm{~h}$ after exposure, we observed that treatment with CAM833 causes an increase in the percentage of cells with 4N DNA 4 hours after irradiation. Over time, there is a drop in the percentage of cells with 4N DNA in both treated and control groups. However, whereas in the control the percentage of cells in the apoptotic subG1 fraction remains below $5 \%$ throughout, in the compound-treated cells this rises progressively to peak at $15 \%$ at 48 hours (Figure 5D). Thus, these results suggest that treatment with CAM833 in- creases the progression of $\mathrm{G} 2 / \mathrm{M}$-arrested cells into apoptosis, as opposed to recovery.

CAM833 causes a dose-dependent growth inhibition that is enhanced when combined with ionizing radiation Consistent with these results, we find that CAM833 suppresses, in a concentration-dependent manner, the growth of multiple cancer-derived human cell lines (Table S2). For instance, CAM833 alone inhibits the growth of HCT116 colon carcinoma cells with an average $50 \%$ growth inhibition $\left(\mathrm{GI}_{50}\right)$ value of $38 \mu \mathrm{M}$ (geometrical mean, $\mathrm{n}=18$, SD $6.6 \mu \mathrm{M}$ ) after $96 \mathrm{~h}$ exposure. Moreover, our results suggest that CAM833 enhances cellular sensitivity to agents such as IR that induce DNA breakage normally repaired through RAD51-dependent HDR. Thus, when combined with 3 Gy IR, CAM833 suppresses the growth of HCT116 cells with a $\mathrm{Gl}_{50}$ of $14 \mu \mathrm{M}$ (geometrical mean, $\mathrm{n}=18, \mathrm{SD}, 6.2 \mu \mathrm{M})$, a concentration more than 2-fold lower than the $\mathrm{GI}_{50}$ for CAM833 alone (Figure 5E).

These findings prompted us to compare the effects of CAM833 with those of Carboplatin, a DNA cross-linking agent used in the clinic to sensitize cancers to therapeutic radiation (Clamon et al., 1999). We first exposed cells to a fixed $10 \mu \mathrm{M}$ dose of either CAM833 or carboplatin, before treatment with 0 to 3 Gy IR, and compared cell growth using the sulforhodamine B cell proliferation assay $96 \mathrm{~h}$ afterwards (Figure 6A). Whereas carboplatin alone is more growth-suppressive than CAM833 alone, combination with increasing doses of IR potentiates the effects of CAM833 but not carboplatin (Figure 6A). The concentration-response curves (Figures $6 \mathrm{~B}$ and $6 \mathrm{C}$ ) showing the effect of combining 0 to 3 Gy IR with different concentrations of either carboplatin or CAM833 reflect a complex, dose-dependent response to the combined effects of CAM833 with IR, leading to changes in the observed IC ${ }_{50}$ (Figure 6D). IR at 1 to 2 Gy sharply potentiates the growth-inhibitory effects of $5 \times 10^{-5}$ to $5 \times 10^{-4} \mathrm{M}$ doses of CAM833. IR at $3 \mathrm{~Gy}$ has a smaller effect, across a wider dose range of CAM833. These differences could arise from biological factors such as variations in the amount or type of IR-induced DNA lesions, and/or the relative contribution of HDR to their repair. Collectively, these findings suggest the potential utility of CAM833 as a radio sensitizer.

\section{CAM833 potentiates PARP1 inhibition in cells wild-type for BRCA2}

Cells deficient in RAD51-mediated HDR through the inactivation of tumor suppressor genes like BRCA1 or BRCA2 exhibit hypersensitivity to poly-ADP ribose polymerase 1 (PARP1) inhibitors (Bryant et al., 2005; Farmer et al., 2005). We therefore tested whether CAM833 could potentiate the growth inhibitory effects of PARP1 inhibition by the inhibitor AZD2461 (Jaspers et al., 2013) in cells wild-type for BRCA2 (Figure 7). To this end, we

(B) Distribution of the number of RAD51 molecules contained within damage-induced clusters in EUFA423 or EUFA423 + BRCA2 cells, without or with exposure to $25 \mu \mathrm{M}$ CAM833, $3 \mathrm{~h}$ after exposure to $3 \mathrm{~Gy}$ ionizing radiation. The box plot was generated using the Matlab boxplot function. The central mark indicates the median (purple dot). The bottom and top edges of the box indicate the 25th and 75th percentiles, respectively. The whiskers extend to the most extreme data points not considered outliers. The mean is marked by a purple line. ${ }^{* \star}$ and $n$.s. indicates $p$ values lower than $10^{-5}$ and not significant differences, respectively. (C) Representative SMLM images of RAD51, represented as 2D Voronoi polygons. The color of the polygons shows molecular densities normalized to the maximum value. Scale bar, $500 \mathrm{~nm}$.

(D) High-magnification SMLM images of damage-induced RAD51 filaments in EUFA423 + BRCA2 cells (DMSO-control left-hand panels), and their suppression by CAM833 (right-hand panels), under the same experimental conditions, at higher magnification. Scale bar, $200 \mathrm{~nm}$. Images are shown either at low-resolution (LR) or super-resolved (SR). Results are representative of two independent repeats. 
A

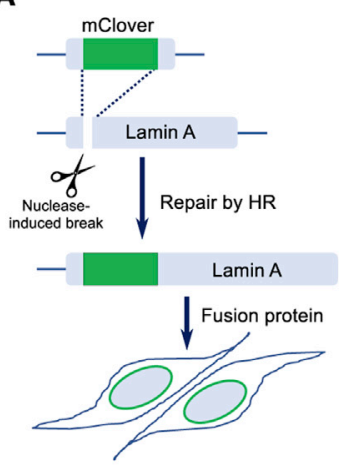

D

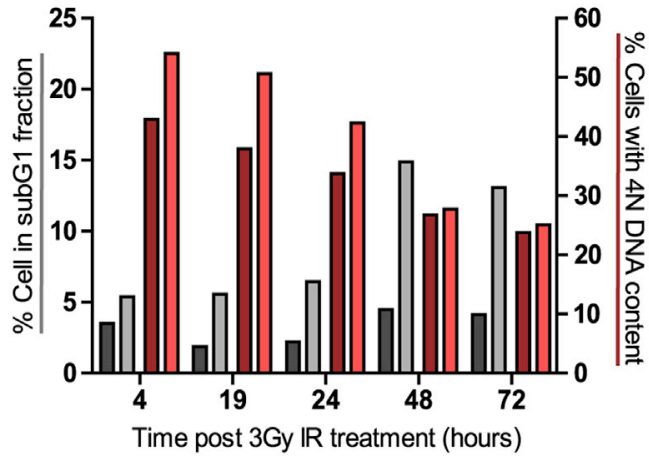

CAM833 dose

B

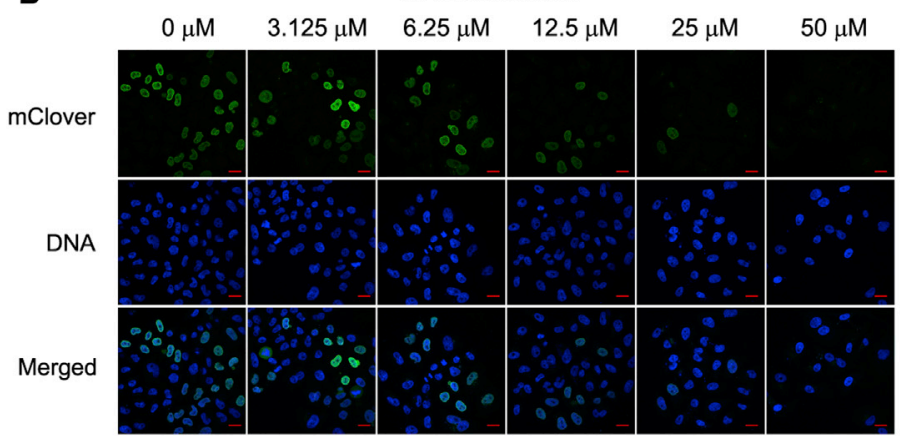

C

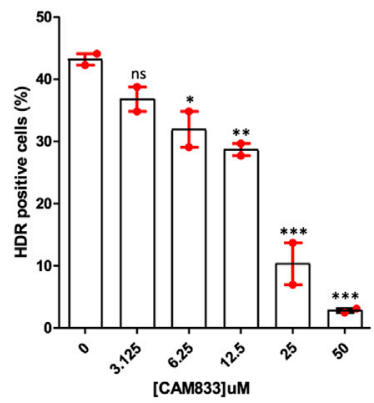

E

SubG1 DMSO control $\square$ SubG1 $20 \mu \mathrm{M}$ CAM833 4N DMSO control $\square$ 4N $20 \mu \mathrm{M}$ CAM833

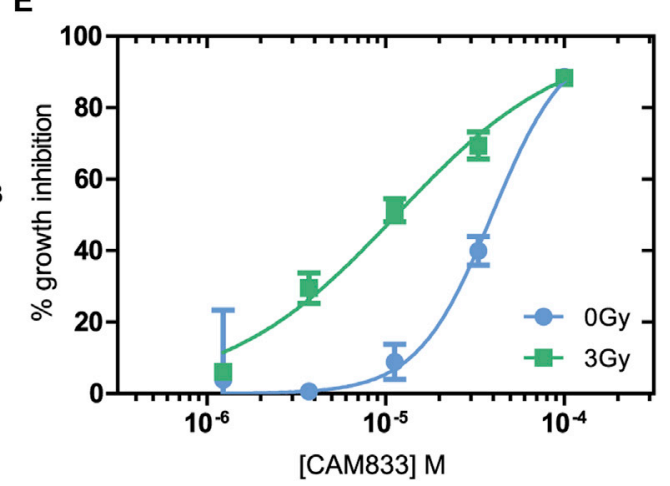

Figure 5. CAM833 inhibits homologous recombination and potentiates cell-cycle arrest

(A) Schematic depiction of the mClover-Lamin assay for DNA repair by homologous DNA recombination (HDR). HeLa Kyoto cells were transfected with the assay plasmids (Lamin A-targeting sgRNA and mClover Lamin A donor constructs) and analyzed for mClover Lamin A-positive (HDR-positive) cells after 3 days. CAM833 was added to the cells $1 \mathrm{~h}$ before transfection and maintained until analysis by microscopy.

(B) Representative microscopic fields showing fluorescence of the mClover-Lamin A fusion protein, DNA (DAPI staining) and merged images after exposure to increasing doses of CAM833. Scale bar, $20 \mu \mathrm{m}$.

(C) Histogram showing the mean of HDR positive cells (\%) \pm SE from two independent repeats. More than 200 cells per sample were analyzed in each repeat. Statistical significance was tested by a 1-way ANOVA test, followed by Dunnett's multiple comparison test: $n s, p$ value $>0.05 ;{ }^{*} p$ value $\leq 0.05$; ${ }^{* *} p$ value $\leq 0.01$; ${ }^{* \star *} \mathrm{p}$ value $\leq 0.001$

(D) Cell cycle analysis of HCT116 cells over a 72-h time course after treatment with $20 \mu \mathrm{M}$ CAM833 or DMSO control, combined with exposure to 3 Gy ionizing radiation.

(E) Dose-response curves for growth inhibition of HCT-116 cells combining 0 (blue circles) or 3 Gy (green squares) of IR at different doses of CAM833. Cell growth was measured after $96 \mathrm{~h}$ using the sulforhodamine B cell proliferation assay. Each plotted value represents the mean percent growth inhibition \pm SEM compared with control cells exposed to DMSO plus the indicated IR dose.

determined dose-response curves for growth inhibition in cells exposed to different doses of AZD2461 combined with a fixed dose of either $10 \mu \mathrm{M}$ (Figure $7 \mathrm{~A}$ ) or $20 \mu \mathrm{M}$ (Figure $7 \mathrm{~B}$ ) of CAM833. While CAM833 alone had little effect (blue triangles), its combination with AZD2461 potentiated the growth-suppressive effects of PARP1 inhibition in a dose-dependent manner. Reciprocally, we also measured the dose-response curves for growth inhibition in cells exposed to different doses of CAM833 combined with a fixed dose of either $0.1 \mu \mathrm{M}$ (Figure $7 \mathrm{C}$ ) or $1 \mu \mathrm{M}$ (Figure 7D) of AZD2461. These doses of AZD2461 have little effect when administered alone (blue diamonds), but again, their combination with CAM833 potentiates growth suppression by PARP1 inhibition in cells wild-type for BRCA2.

\section{DISCUSSION}

We report here the discovery of CAM833, a sub-micromolar chemical inhibitor of the regulatory protein-protein interaction between the RAD51 recombinase and the BRC repeat motifs of the tumor suppressor BRCA2. Using structure determination by X-ray crystallography, we show that CAM833 engages with two hydrophobic pockets on the surface of RAD51 that normally accommodate conserved hydrophobic side chains from the BRC repeats of BRCA2, thereby directly competing with the RAD51:BRCA2 interaction. These pockets also normally mediate RAD51 multimerization on DNA substrates during the process that leads to HDR, by accommodating corresponding hydrophobic residues from an adjacent RAD51 protomer to form the protomer-protomer interface. Consistent with these structural considerations, we show that CAM833 suppresses the assembly of RAD51 into damage-induced filaments visualized by single-molecule localization microscopy, and impairs DNA repair by homologous DNA recombination. Moreover, we present multiple lines of evidence suggesting that CAM833 potentiates growth inhibition, cell-cycle arrest and cytotoxicity induced by DNA damage, consistent with its predicted ability 
A

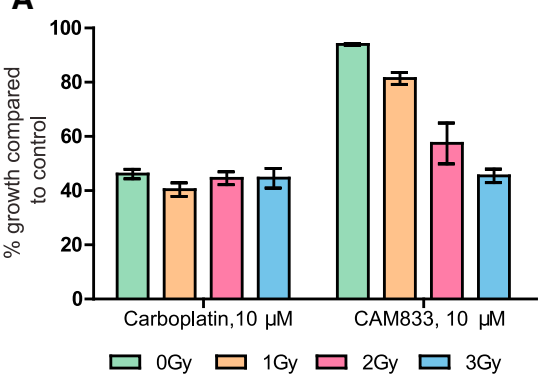

B

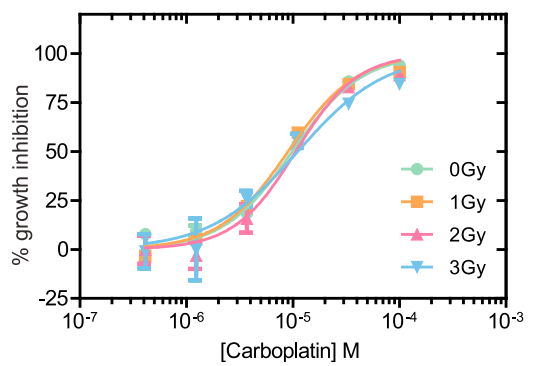

C

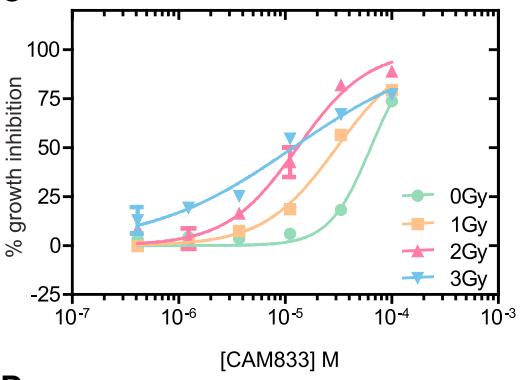

D

Carboplatin
\begin{tabular}{|c|c|c|c|c|}
\hline Gy & 0 & 1 & 2 & 3 \\
\hline IC50 & 9.9 & 9.0 & 10.6 & 11.1 \\
\hline
\end{tabular}

Figure 6. Low-dose ionizing radiation potentiates the effects of CAM833 but not carboplatin

(A) Cell growth after exposure to increasing levels of ionizing radiation in the presence of a fixed dose $(10 \mu \mathrm{M})$ of either Carboplatin or CAM833. Bars depict percent growth compared with control cells exposed to DMSO plus the indicated IR dose, represented as the mean \pm SEM. Values $<100$ indicate growth inhibition.

(B and C) Plot dose-response curves for growth inhibition combining 0 (green circles), 1 Gy (orange squares), 2 Gy (red triangles), or 3 Gy (blue triangles) of IR with different doses of carboplatin (B) or CAM833 (C) shown as molar. In (B) and (C), each plotted value represents the mean percent growth inhibition \pm SEM compared with control cells exposed to DMSO plus the indicated IR dose.

(D) Observed changes in $\mathrm{IC}_{50}$ (expressed in $\mu \mathrm{M}$ ) for growth inhibition derived from the curves in $(B)$ and (C). These data are representative of 3 independent experiments.

to suppress DNA repair by HDR. Our findings have several important implications.

CAM833 is a well-characterized, selective chemical probe molecule that should prove valuable for further elucidating the biology of the RAD51-BRCA2 protein-protein interaction and the associated HDR pathways. Moreover, CAM833 is a chemically tractable starting point for the further, structure-guided development of optimized inhibitory compounds with the potential for development into a drug compound suitable for clinical studies. The development of this molecule through an innovative strategy of combining a fragment hit with a peptide lead compound reveals what is likely to be a generally applicable strategy for the development of inhibitors of protein-protein interactions featuring a continuous peptide epitope (Scott et al., 2016).

Our work exemplifies a strategy to modulate the activity of RAD51 during HDR through two of its key regulatory protein-protein interactions. The first of these interactions is between RAD51 and the BRC repeats of BRCA2, which is essential to target RAD51 to cellular sites of DNA damage, and may also regulate RAD51 assembly on DNA substrates at these sites. The second interaction blocked by CAM833 is between RAD51 protomers, which occurs at the same structural motif engaged by the BRC repeats, and enables RAD51 assembly by multimerization. Our findings provide several lines of evidence that
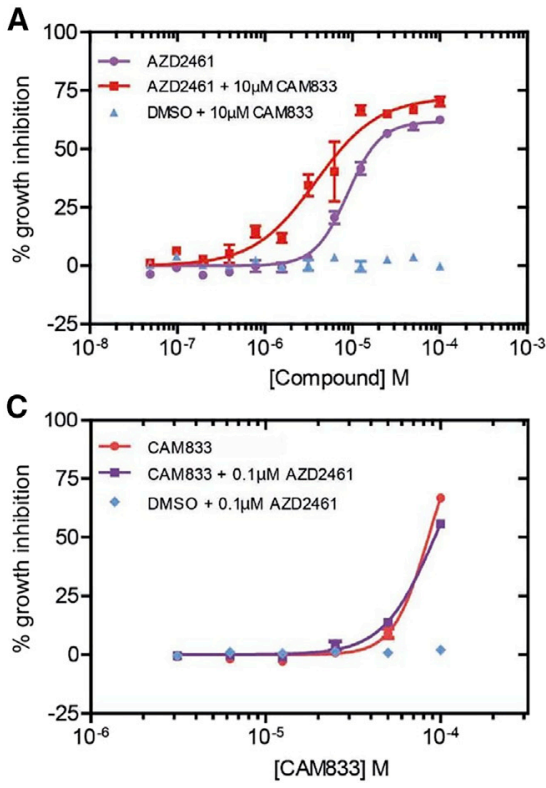
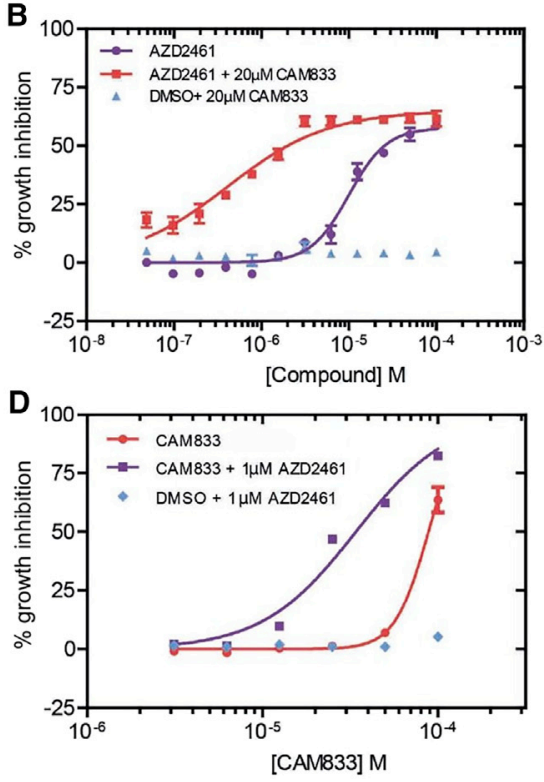

Figure 7. CAM833 potentiates the growth suppressive effect of PARP1 inhibition in BRCA2 wild-type cells

(A and B) Dose-response curves for growth inhibition in HCT116 cells exposed to different doses of AZD2461 plotted as molar combined with a fixed dose of either $10 \mu \mathrm{M}(\mathrm{A})$ or $20 \mu \mathrm{M}$ (B) of CAM833. Control experiments in which vehicle (DMSO) was added in place of AZD2461 are plotted in blue. Growth was measured $96 \mathrm{~h}$ after compound exposure using the SRB assay, and is depicted as the mean percent inhibition \pm SEM compared with controls.

(C and D) Reciprocal dose-response curves for growth inhibition after exposure to different doses of CAM833 plotted as molar combined with a fixed dose of either $0.1 \mu \mathrm{M}(\mathrm{C})$ or $1 \mu \mathrm{M}$ (D) of AZD2461. Control experiments in which vehicle (DMSO) was added in place of CAM833 are plotted in blue. Measurements and plots are as in the previous panels. Results are representative of two independent repeats. 
CAM833 acts in cells to engage RAD51 and block the proteinprotein interactions that lead to its multimerization at sites of DNA damage. We find using SMLM by d-STORM that CAM833 suppresses the molecular clustering of RAD51 at damage sites, and prevents the extension of these clusters into extended RAD51 filaments, providing evidence for target engagement and the proposed mechanism of action. The mechanism of CAM833 action via the inhibition of RAD51-mediated HDR is further supported by our finding that the compound sensitizes cells with wild-type BRCA2 to the growth inhibitory effects of the PARP1 inhibitor, AZD2461. In the context of wild-type BRCA2, PARP1 inhibition alone is usually ineffective. While these results further support the cellular mechanism underlying CAM833 action, we are skeptical that systemic inhibition of RAD51 combined with the systemic effects of PARP1 inhibition has therapeutic potential owing to the likelihood of dose-limiting mechanism-related toxicity in normal tissues.

However, CAM833 also potentiates the cellular effects of ionizing radiation, a potent inducer of DNA breakage. When combined with IR, CAM833 sensitizes cells to IR-induced cellcycle arrest at the G2/M phase of the cell cycle, and enhances cell death by apoptosis. Collectively, these findings provide evidence supporting the further development of small-molecule inhibitors of the regulatory protein-protein interactions of RAD51 for cancer therapy through radiosensitisation. Indeed, the locoregional nature of radiation therapy may prove in this regard to have a higher therapeutic index.

\section{SIGNIFICANCE}

Protein-protein interactions that mediate intracellular reactions leading to the repair of damaged DNA are an important target for anti-cancer drug discovery. Here, we report using structure-guided lead discovery the development of a potent orthosteric inhibitor, CAM833, of the protein-protein interaction between the BRCA2 tumor suppressor and the RAD51 recombinase, which is critical for the error-free repair of DNA breakage by homologous DNA recombination. The significance of our work is 3-fold. First, it exemplifies a strategy for the development of inhibitors that target protein-protein interactions wherein a contiguous series of amino acids interact with a protein surface, by merging a peptidic inhibitor derived from those amino acids with chemical fragment hits identified by biophysical and crystallographic screening. Second, we demonstrate using singlemolecule localization ("super-resolution") microscopy that CAM833 inhibits RAD51 molecular clustering to prevent the assembly of extended RAD51 filaments at sites of DNA damage, validating target engagement, and demonstrating a unique mechanism of action. Finally, we show that CAM833 inhibits the cellular response to DNA damage, potentiating in BRCA2 wild-type cells the cytotoxic effects both of ionizing radiation or of PARP1 inhibitors, opening future avenues for anti-cancer drug development.

\section{STAR $\star$ METHODS}

Detailed methods are provided in the online version of this paper and include the following:
- KEY RESOURCES TABLE

- RESOURCE AVAILABILITY

O Lead contact

O Materials availability

O Data and code availability

- EXPERIMENTAL MODEL AND SUBJECT DETAILS

O E. coli for protein production

O Cell line and cell culture

- METHOD DETAILS

O Chemical synthesis

Protein production

O ITC

FP assay

X-ray crystallography

O Immunofluorescent visualisation of RAD51 foci $/ \gamma \mathrm{H} 2 \mathrm{AX}$ foci in A549 cells using the Cellomics Arrayscan $\mathrm{V}^{\mathrm{ti}}$ high content microscopy

O SRB growth inhibition assay

O Flow cytometry

O Super-resolution microscopy

O Cluster data analysis

O mClover Lamin A HDR assay

- QUANTIFICATION AND STATISTICAL ANALYSES

\section{SUPPLEMENTAL INFORMATION}

Supplemental Information can be found online at https://doi.org/10.1016/j. chembiol.2021.02.006.

\section{ACKNOWLEDGMENTS:}

We thank Dr. Adrian Schreyer for developing a database for data management and Dr. Tara Pukala and Prof. Carol Robinson for taking part in the early stages of this project. We are grateful for Diamond Light Source for access to beamlines I02, 104 and 124 (proposals $m \times 315$ and $m \times 7141$ ) and ESRF for access beamline ID14-4. We thank the X-ray crystallographic and biophysics facilities at the Department of Biochemistry for support and access. This work was funded by the Wellcome Trust Translational Award (080083/Z/06/Z) and Seeding Drug Discovery Award (091058/Z/ 09/Z) and we acknowledge the support of their Seeding Drug Discovery team, in particularly Dr. Sarah Hardy and Prof. Chas Bountra, for useful discussions. This work was also funded by Medical Research Council (MRC) Program grants MC_UU_12022/1 and MC_UU_12022/8 to ARV, and a research grant from Astex Pharmaceuticals. We thank WuXi AppTec for Pharmacokinetic and cell-line sensitivity data and Cyprotex for ADMET data. C.A., senior author of this paper and a good friend and colleague of the remaining authors, sadly died while this manuscript was in revision; we dedicate this paper to him.

\section{AUTHOR CONTRIBUTIONS}

Experimental investigations in Figures 1 and 2 were carried out by D.E.S., T.P.S., A.G.C., C.D., G.F., C.V., T.M., A.R.B., M.E.M., R.S., D.H., A.H., M.E. T.P., and J.S.; in Figures 3, 5, 6, and 7, by N.J.F.-N. and L.B.; in Figure 4, by K.T.H. and A.E.; and in Figure 5 by M.L., A.E., and B.H. T.L.B., A.R.V., C.A., M.H., L.P., G.M., J.S., and T.P. conceptualized the project, supervised the experimental work, and analyzed the results. D.E.S., N.J.F.-N., J.S., M.H., C.A., and A.R.V. wrote the manuscript with input from all the authors. Each corresponding author supervised and is responsible for distinct aspects of this multidisciplinary project. Chemistry was led by J.S. and C.A.; biochemistry and structural biology by M.H.; and microscopy, cell genetics, and cell biology by A.R.V. 


\section{CellPress}

\section{DECLARATION OF INTERESTS}

Venkitaraman, Pellegrini, Blundell et al., WO2004035621 - Use of crystal structure of human RAD51-BRCA2 repeat complex in screening for anti tumor agents.

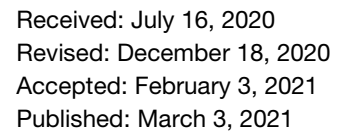

\section{REFERENCES}

Adams, P.D., Afonine, P.V., Bunkóczi, G., Chen, V.B., Davis, I.W., Echols, N., Headd, J.J., Hung, L.-W., Kapral, G.J., Grosse-Kunstleve, R.W., et al. (2010). PHENIX: a comprehensive Python-based system for macromolecular structure solution. Acta Crystallogr. D. Biol. Crystallogr. 66, 213-221.

Arnoult, N., Correia, A., Ma, J., Merlo, A., Garcia-Gomez, S., Maric, M., Tognetti, M., Benner, C.W., Boulton, S.J., Saghatelian, A., et al. (2017). Regulation of DNA repair pathway choice in S and G2 phases by the NHEJ inhibitor CYREN. Nature 549, 548-552.

Bagnolini, G., Milano, D., Manerba, M., Schipani, F., Ortega, J.A., Gioia, D., Falchi, F., Balboni, A., Farabegoli, F., De Franco, F., et al. (2020). Synthetic lethality in pancreatic cancer: discovery of a new RAD51-BRCA2 small molecule disruptor that inhibits homologous recombination and synergizes with olaparib. J. Med. Chem. 63, 2588-2619.

Bignell, G., Micklem, G., Stratton, M.R., Ashworth, A., and Wooster, R. (1997). The BRC repeats are conserved in mammalian BRCA2 proteins. Hum. Mol. Genet. 6, 53-58.

Blundell, T.L., Jhoti, H., and Abell, C. (2002). High-throughput crystallography for lead discovery in drug design. Nat. Rev. Drug Discov. 1, 45-54.

Brouwer, I., Moschetti, T., Candelli, A., Garcin, E.B., Modesti, M., Pellegrini, L., Wuite, G.J., and Peterman, E.J. (2018). Two distinct conformational states define the interaction of human RAD51- ATP with single-stranded DNA. EMBO J. 37, e98162.

Bryant, H.E., Schultz, N., Thomas, H.D., Parker, K.M., Flower, D., Lopez, E., Kyle, S., Meuth, M., Curtin, N.J., and Helleday, T. (2005). Specific killing of BRCA2-deficient tumours with inhibitors of poly(ADP-ribose) polymerase. Nature 434, 913-917.

Budke, B., Kalin, J.H., Pawlowski, M., Zelivianskaia, A.S., Wu, M., Kozikowski, A.P., and Connell, P.P. (2012a). An optimized RAD51 inhibitor that disrupts homologous recombination without requiring Michael acceptor reactivity. J. Med. Chem. 56, 254-263.

Budke, B., Logan, H.L., Kalin, J.H., Zelivianskaia, A.S., Cameron McGuire, W., Miller, L.L., Stark, J.M., Kozikowski, A.P., Bishop, D.K., and Connell, P.P. (2012b). RI-1: a chemical inhibitor of RAD51 that disrupts homologous recombination in human cells. Nucleic Acids Res. 40, 7347-7357.

Budke, B., Tueckmantel, W., Miles, K., Kozikowski, A.P., and Connell, P.P. (2019). Optimization of drug candidates that inhibit the D-loop activity of RAD51. ChemMedChem 14, 1031-1040.

Buisson, R., Niraj, J., Rodrigue, A., Kwen Ho, C., Krauzer, J., Foo, T.K., Hardy, E.L.-J., Dellaire, G., Hass, W., Xia, B., et al. (2017). Coupling of homologous recombination and the checkpoint by ATR. Mol. Cell 65, 336-346.

Carreira, A., and Kowalczykowski, S.C. (2011). Two classes of BRC repeats in BRCA2 promote RAD51 nucleoprotein filament function by distinct mechanisms. Proc. Natl. Acad. Sci. U S A 108, 10448-10453.

Carreira, A., Hilario, J., Amitani, I., Baskin, R.J., Shivji, M.K.K., Venkitaraman, A.R., and Kowalczykowski, S.C. (2009). The BRC repeats of BRCA2 modulate the DNA-binding selectivity of RAD51. Cell 136, 1032-1043.

Chen, C.-F., Chen, P.-L., Zhong, Q., Sharp, Z.D., and Lee, W.-H. (1999a). Expression of BRC repeats in breast cancer cells disrupts the BRCA2Rad51 complex and leads to radiation hypersensitivity and loss of $G$ 2/M checkpoint control. J. Biol. Chem. 274, 32931-32935.

Chen, G., Yuan, S.S.F., Liu, W., Xu, Y., Trujillo, K., Song, B., Cong, F., Goff, S.P., Wu, Y., Arlinghaus, R., et al. (1999b). Radiation-induced assembly of
Cell Chemical Biology Article

Rad51 and Rad52 recombination complex requires ATM and c-Abl. J. Biol. Chem. 274, 12748-12752.

Clamon, G., Herndon, J., Cooper, R., Chang, A.Y., Rosenman, J., and Green, M.R. (1999). Radiosensitization with carboplatin for patients with unresectable stage III non-small-cell lung cancer: a phase III trial of the Cancer and Leukemia Group B and the Eastern Cooperative Oncology Group. J. Clin. Oncol. 17, 4.

Cole, D.J., Janecek, M., Stokes, J.E., Rossmann, M., Faver, J.C., McKenzie, G.J., Venkitaraman, A.R., Hyvönen, M., Spring, D.R., Huggins, D.J., et al. (2017). Computationally-guided optimization of small-molecule inhibitors of the Aurora A kinase-TPX2 protein-protein interaction. Chem. Commun. 53, 9372-9375.

Conway, A.B., Lynch, T.W., Zhang, Y., Fortin, G.S., Fung, C.W., Symington, L.S., and Rice, P.A. (2004). Crystal structure of a Rad51 filament. Nat. Struct. Mol. Biol. 11, 791-796.

Coyne, A.G., Scott, D.E., and Abell, C. (2010). Drugging challenging targets using fragment-based approaches. Curr. Opin. Chem. Biol. 14, 299-307.

Davies, O.R., and Pellegrini, L. (2007). Interaction with the BRCA2 C terminus protects RAD51-DNA filaments from disassembly by BRC repeats. Nat. Struct. Mol. Biol. 14, 475-483.

Davies, A.A., Masson, J.Y., Mcllwraith, M.J., Stasiak, A.Z., Stasiak, A., Venkitaraman, A.R., and West, S.C. (2001). Role of BRCA2 in control of the RAD51 recombination and DNA repair protein. Mol. Cell 7, 273-282.

Esashi, F., Galkin, V.E., Yu, X., Egelman, E.H., and West, S.C. (2007). Stabilization of RAD51 nucleoprotein filaments by the C-terminal region of BRCA2. Nat. Struct. Mol. Biol. 14, 468-474.

Falchi, F., Giacomini, E., Masini, T., Boutard, N., Di lanni, L., Manerba, M., Farabegoli, F., Rossini, L., Robertson, J., Minucci, S., et al. (2017). Synthetic lethality triggered by combining olaparib with BRCA2-Rad51 disruptors. ACS Chem. Biol. 12, 2491-2497.

Farmer, H., McCabe, N., Lord, C.J., Tutt, A.N.J., Johnson, D.A., Richardson, T.B., Santarosa, M., Dillon, K.J., Hickson, I., Knights, C., et al. (2005). Targeting the DNA repair defect in BRCA mutant cells as a therapeutic strategy. Nature 434, 917-921.

Haas, K.T., Lee, M., Esposito, A., and Venkitaraman, A.R. (2018). Single-molecule localization microscopy reveals molecular transactions during RAD51 filament assembly at cellular DNA damage sites. Nucleic Acids Res. 46, 2398-2416.

Hattori, H., Skoulidis, F., Russell, P., and Venkitaraman, A.R. (2011). Context dependence of checkpoint kinase 1 as a therapeutic target for pancreatic cancers deficient in the BRCA2 tumor suppressor. Mol. Cancer Ther. 10, 670-678.

Howlett, N.G., Taniguchi, T., Olson, S., Cox, B., Waisfisz, Q., De Die-Smulders, C., Persky, N., Grompe, M., Joenje, H., Pals, G., et al. (2002). Biallelic inactivation of BRCA2 in Fanconi anemia. Science 297, 606-609.

Huang, F., and Mazin, A.V. (2014). A small molecule inhibitor of human RAD51 potentiates breast cancer cell killing by therapeutic agents in mouse xenografts. PLoS One 9, e100993.

Huang, F., Motlekar, N.A., Burgwin, C.M., Napper, A.D., Diamond, S.L., and Mazin, A.V. (2011). Identification of specific inhibitors of human RAD51 recombinase using high-throughput screening. ACS Chem. Biol. 6, 628-635.

Ishida, T., Takizawa, Y., Kainuma, T., Inoue, J., Mikawa, T., Shibata, T., Suzuki, H., Tashiro, S., and Kurumizaka, H. (2009). DIDS, a chemical compound that inhibits RAD51-mediated homologous pairing and strand exchange. Nucleic Acids Res. 37, 3367-3376.

Jaspers, J.E., Kersbergen, A., Boon, U., Sol, W., van Deemter, L., Zander, S.A., Drost, R., Wientjens, E., Ji, J., Aly, A., et al. (2013). Loss of 53BP1 causes PARP inhibitor resistance in Brca1 -mutated mouse mammary tumors. Cancer Discov. 3, 68-81.

Jeyasekharan, A.D., Liu, Y., Hattori, H., Pisupati, V., Jonsdottir, A.B., Rajendra, E., Lee, M., Sundaramoorthy, E., Schlachter, S., Kaminski, C.F., et al. (2013). A cancer-associated BRCA2 mutation reveals masked nuclear export signals controlling localization. Nat. Struct. Mol. Biol. 20, 1191-1198.

Kabsch, W. (2010). XDS. Acta Crystallogr. D Biol. Crystallogr. 66, 125-132. 
Lv, W., Budke, B., Pawlowski, M., Connell, P.P., and Kozikowski, A.P. (2016). Development of small molecules that specifically inhibit the D-loop activity of RAD51. J. Med. Chem. 59, 4511-4525.

Moschetti, T., Sharpe, T., Fischer, G., Marsh, M.E., Ng, H.K., Morgan, M., Scott, D.E., Blundell, T.L., R Venkitaraman, A., Skidmore, J., et al. (2016). Engineering archeal surrogate systems for the development of proteinprotein interaction inhibitors against human RAD51. J. Mol. Biol. 428, 4589-4607.

Nomme, J., Renodon-Cornière, A., Asanomi, Y., Sakaguchi, K., Stasiak, A.Z., Stasiak, A., Norden, B., Tran, V., and Takahashi, M. (2010). Design of potent inhibitors of human RAD51 recombinase based on BRC motifs of BRCA2 protein: modeling and experimental validation of a chimera peptide. J. Med. Chem. 53, 5782-5791.

Normand, A., Rivière, E., and Renodon-Cornière, A. (2014). Identification and characterization of human Rad51 inhibitors by screening of an existing drug library. Biochem. Pharmacol. 91, 293-300.

Pastushok, L., Fu, Y., Lin, L., Luo, Y., DeCoteau, J.F., Lee, K., and Geyer, C.R. (2019). A novel cell-penetrating antibody fragment inhibits the DNA repair protein RAD51. Sci. Rep. 9, 11227.

Pellegrini, L., Yu, D.S., Lo, T., Anand, S., Lee, M., Blundell, T.L., and Venkitaraman, A.R. (2002). Insights into DNA recombination from the structure of a RAD51-BRCA2 complex. Nature 420, 287-293.

Rajendra, E., and Venkitaraman, A.R. (2010). Two modules in the BRC repeats of BRCA2 mediate structural and functional interactions with the RAD51 recombinase. Nucleic Acids Res. 38, 82-96.

Roberti, M., Schipani, F., Bagnolini, G., Milano, D., Giacomini, E., Falchi, F., Balboni, A., Manerba, M., Farabegoli, F., De Franco, F., et al. (2019). Rad51/ BRCA2 disruptors inhibit homologous recombination and synergize with olaparib in pancreatic cancer cells. Eur. J. Med. Chem. 165, 80-92.

Rogakou, E.P., Pilch, D.R., Orr, A.H., Ivanova, V.S., and Bonner, W.M. (1998). DNA double-stranded breaks induce histone H2AX phosphorylation on serine 139. J. Biol. Chem. 273, 5858-5868.

Schneider, C., Rasband, W., and Eliceiri, K. (2012). NIH Image to ImageJ: 25 years of image analysis. Nat Methods 9, 671-675.

Scott, D.E., Ehebauer, M.T., Pukala, T., Marsh, M., Blundell, T.L., Venkitaraman, A.R., Abell, C., and Hyvönen, M. (2013). Using a fragmentbased approach to target protein-protein interactions. Chembiochem 14, 332-342.

Scott, D.E., Marsh, M., Blundell, T.L., Abell, C., and Hyvönen, M. (2016). Structure-activity relationship of the peptide binding-motif mediating the BRCA2:RAD51 protein-protein interaction. FEBS Lett. 590, 1094-1102.

Shin, D.S., Pellegrini, L., Daniels, D.S., Yelent, B., Craig, L., Bates, D., Yu, D.S., Shivji, M.K., Hitomi, C., Arvai, A.S., et al. (2003). Full-length archaeal Rad51 structure and mutants: mechanisms for RAD51 assembly and control by BRCA2. EMBO J. 22, 4566-4576.

Shivji, M.K.K., Mukund, S.R., Rajendra, E., Chen, S., Short, J.M., Savill, J., Klenerman, D., and Venkitaraman, A.R. (2009). The BRC repeats of human BRCA2 differentially regulate RAD51 binding on single- versus doublestranded DNA to stimulate strand exchange. Proc. Natl. Acad. Sci. U S A 106, 13254-13259.

Short, J.M., Liu, Y., Chen, S., Soni, N., Madhusudhan, M.S., Shivji, M.K.K., and Venkitaraman, A.R. (2016). High-resolution structure of the presynaptic RAD51 filament on single-stranded DNA by electron cryo-microscopy. Nucleic Acids Res. 44, 9017-9030.

Sonoda, E., Sasaki, M.S., Buerstedde, J.M., Bezzubova, O., Shinohara, A., Ogawa, H., Takata, M., Yamaguchi-Iwai, Y., and Takeda, S. (1998). Rad51deficient vertebrate cells accumulate chromosomal breaks prior to cell death. EMBO J. 17, 598-608.

Su, X., Bernal, J.A., and Venkitaraman, A.R. (2008). Cell-cycle coordination between DNA replication and recombination revealed by a vertebrate $\mathrm{N}$-end rule degron-Rad51. Nat. Struct. Mol. Biol. 15, 1049-1058.

Takaku, M., Kainuma, T., Ishida-Takaku, T., Ishigami, S., Suzuki, H., Tashiro, S., van Soest, R.W.M., Nakao, Y., and Kurumizaka, H. (2011). Halenaquinone, a chemical compound that specifically inhibits the secondary DNA binding of RAD51. Genes Cells 16, 427-436.

Trenner, A., Godau, J., and Sartori, A.A. (2018). A short BRCA2-derived cellpenetrating peptide targets RAD51 function and confers hypersensitivity toward PARP inhibition. Mol. Cancer Ther. 17, 1392-1405.

Turchick, A., Hegan, D.C., Jensen, R.B., and Glazer, P.M. (2017). A cell-penetrating antibody inhibits human RAD51 via direct binding. Nucleic Acids Res. 45, 11782-11799.

Turchick, A., Liu, Y., Zhao, W., Cohen, I., and Glazer, P.M. (2019). Synthetic lethality of a cell-penetrating anti-RAD51 antibody in PTEN-deficient melanoma and glioma cells. Oncotarget 10, 1272-1283.

Venkitaraman, A.R. (2014). Tumour suppressor mechanisms in the control of chromosome stability: insights from BRCA2. Mol. Cells 37, 95-99.

Vonrhein, C., Flensburg, C., Keller, P., Sharff, A., Smart, O., Paciorek, W., Womack, T., and Bricogne, G. (2011). Data processing and analysis with the autoPROC toolbox. Acta Crystallogr. D Biol. Crystallogr. 67, 293-302.

Vydyam, P., Dutta, D., Sutram, N., Bhattacharyya, S., and Bhattacharyya, M.K. (2019). A small-molecule inhibitor of the DNA recombinase Rad51 from Plasmodium falciparum synergizes with the antimalarial drugs artemisinin and chloroquine. J. Biol. Chem. 294, 8171-8183.

Wang, Z.X. (1995). An exact mathematical expression for describing competitive binding of two different ligands to a protein molecule. FEBS Lett. 360, $111-114$.

Ward, A., Dong, L., Harris, J.M., Khanna, K.K., Al-Ejeh, F., Fairlie, D.P., Wiegmans, A.P., and Liu, L. (2017). Quinazolinone derivatives as inhibitors of homologous recombinase RAD51. Bioorg. Med. Chem. Lett. 27, 3096-3100. West, S.C. (2003). Molecular views of recombination proteins and their control. Nat. Rev. Mol. Cell Biol. 4, 435-445.

Wong, A.K.C., Pero, R., Ormonde, P.A., Tavtigian, S.V., and Bartel, P.L. (1997). RAD51 interacts with the evolutionarily conserved BRC motifs in the human breast cancer susceptibility gene brca2. J. Biol. Chem. 272, 31941-31944.

Xu, J., Zhao, L., Xu, Y., Zhao, W., Sung, P., and Wang, H. (2017). Cryo-EM structures of human RAD51 recombinase filaments during catalysis of DNAstrand exchange. Nat. Struct. Mol. Biol. 24, 40-46.

Zhu, J., Zhou, L., Wu, G., Konig, H., Lin, X., Li, G., Qiu, X.-L., Chen, C.-F., Hu, C.-M., Goldblatt, E., et al. (2013). A novel small molecule RAD51 inactivator overcomes imatinib-resistance in chronic myeloid leukaemia. EMBO Mol. Med. 5, 353-365.

Zhu, J., Chen, H., Guo, X.E., Qiu, X., Hu, C., Chamberlin, A.R., and Lee, W. (2015). Synthesis, molecular modeling, and biological evaluation of novel RAD51 inhibitors. Eur. J. Med. Chem. 96, 196-208. 


\section{STAR $\star$ METHODS}

\section{KEY RESOURCES TABLE}

\begin{tabular}{|c|c|c|}
\hline REAGENT or RESOURCE & SOURCE & IDENTIFIER \\
\hline \multicolumn{3}{|l|}{ Antibodies } \\
\hline Mouse polyclonal anti-RAD51 Antibody & Abnova & $\begin{array}{l}\text { Cat\# H00005888-B01P; RRID: } \\
\text { AB_1522243 }\end{array}$ \\
\hline $\begin{array}{l}\text { Alexa Fluor } 488 \text { labelled anti-mouse } \\
\text { secondary antibody }\end{array}$ & Invitrogen & Cat\# A11001; RRID: AB_2534069 \\
\hline $\begin{array}{l}\text { Anti-phospho } \gamma \mathrm{H} 2 \mathrm{AX} \text { primary mouse } \\
\text { monoclonal antibody }\end{array}$ & Millipore & Cat\#05-636; RRID: AB_309864 \\
\hline Rabbit anti-RAD51 monoclonal antibody & Abcam & Cat\# ab 133534; RRID: AB_2722613 \\
\hline \multicolumn{3}{|l|}{ Bacterial and virus strains } \\
\hline BL21(DE3) E. coli strain & New England Biolabs & C2527I \\
\hline \multicolumn{3}{|l|}{ Chemicals, Peptides, and Recombinant Proteins } \\
\hline ChimRAD51 protein & Moschetti et al., 2016 & $\mathrm{~N} / \mathrm{A}$ \\
\hline Alexa Fluor 488-labelled BRC4 peptide & Moschetti et al., 2016 & $\mathrm{~N} / \mathrm{A}$ \\
\hline HumRadA1 protein & Moschetti et al., 2016 & $\mathrm{~N} / \mathrm{A}$ \\
\hline HumRadA22F protein & Moschetti et al., 2016 & $\mathrm{~N} / \mathrm{A}$ \\
\hline RAD51 protein & Moschetti et al., 2016 & $\mathrm{~N} / \mathrm{A}$ \\
\hline Tetrapeptide (1) & Scott et al., 2016 & FHTA \\
\hline Inhibitors $4-6$ and CAM833 & $\begin{array}{l}\text { Synthesis from commercially available } \\
\text { materials described in this paper }\end{array}$ & CAM833 \\
\hline AZD2461 & SelleckChem & Cat\#S7029 \\
\hline MEA-HCl & Sigma & M6500 \\
\hline Glucose oxidase & Sigma & G2133 \\
\hline Catalase & Sigma & C100 \\
\hline \multicolumn{3}{|l|}{ Deposited data } \\
\hline $\begin{array}{l}\text { Crystallographic coordinates of Rad51 in } \\
\text { complex with BRC4 repeat }\end{array}$ & Pellegrini et al., 2002 & PDB: 1NOW \\
\hline $\begin{array}{l}\text { HumRadA2 in complex with oligomerization } \\
\text { peptide }\end{array}$ & Moschetti et al., 2016 & PDB: 5NWL \\
\hline HumRadA1 in complex with 2-naphthol & Scott et al., 2013 & PDB: 4B32 \\
\hline $\begin{array}{l}\text { HumRadA1 in complex with FHTA } \\
\text { tetrapeptide }\end{array}$ & Scott et al., 2013 & PDB: 4B3B \\
\hline $\begin{array}{l}\text { Crystallographic coordinates and structure } \\
\text { factors for } 3 \text { in complex with HumRadA1 }\end{array}$ & This paper & PDB: 6TV3 \\
\hline $\begin{array}{l}\text { Crystallographic coordinates and structure } \\
\text { factors for } 4 \text { in complex with HumRadA1 }\end{array}$ & This paper & PDB: 6 TWR \\
\hline $\begin{array}{l}\text { Crystallographic coordinates and structure } \\
\text { factors for } 6 \text { in complex with HumRadA22F }\end{array}$ & This paper & PDB: 6TW4 \\
\hline $\begin{array}{l}\text { Crystallographic coordinates and structure } \\
\text { factors for } 6 \text { in complex with HumRadA33F }\end{array}$ & This paper & PDB: 6XTW \\
\hline $\begin{array}{l}\text { Crystallographic coordinates and structure } \\
\text { factors for CAM833 in complex with } \\
\text { HumRadA22F }\end{array}$ & This paper & PDB: 6 TW9 \\
\hline \multicolumn{3}{|l|}{ Experimental models: cell lines } \\
\hline HCT116 colon carcinoma cells & ATCC & CCL-185 \\
\hline A549 lung adenocarcinoma cells & ATCC & CCL-247 \\
\hline HeLa Kyoto cells & $\begin{array}{l}\text { From the laboratory of Jonathan Pines, } \\
\text { Institute of Cancer Research, London }\end{array}$ & N/A \\
\hline
\end{tabular}




\begin{tabular}{|c|c|c|}
\hline \multicolumn{3}{|l|}{ Continued } \\
\hline REAGENT or RESOURCE & SOURCE & IDENTIFIER \\
\hline EUFA423 cells & $\begin{array}{l}\text { From the European Fanconi Anemia } \\
\text { Registry, VU University Medical Center, } \\
\text { Amsterdam, The Netherlands }\end{array}$ & $\mathrm{N} / \mathrm{A}$ \\
\hline EUFA423+BRCA2 & Hattori et al., 2011 & $\mathrm{~N} / \mathrm{A}$ \\
\hline \multicolumn{3}{|l|}{ Recombinant DNA } \\
\hline pUC CBA-SpCas9.EF1a-BFP.sgLMNA & Arnoult et al., 2017 & Addgene Plasmid \#98971 \\
\hline pCAGGS Donor mClover-LMNA & Arnoult et al., 2017 & Addgene Plasmid \#98970 \\
\hline $\begin{array}{l}\text { Plasmids for expression of HumRadA and } \\
\text { ChimRAD51 proteins and BRC4 repeat }\end{array}$ & Moschetti et al., 2016 & N/A \\
\hline \multicolumn{3}{|l|}{ Software and algorithms } \\
\hline Origin for ITC200 & Malvern Instruments & https://www.malvernpanalytical.com/en \\
\hline XDS & MPI for Medical Research & http://xds.mpimf-heidelberg.mpg.de/ \\
\hline Autoproc & Global Phasing & https://www.globalphasing.com/autoproc/ \\
\hline Phenix.refine & Phenix consortium & https://www.phenix-online.org/ \\
\hline autoBuster & Global Phasing & https://www.globalphasing.com/buster/ \\
\hline Prism & GraphPad & $\begin{array}{l}\text { https://www.graphpad.com/scientific- } \\
\text { software/prism/ }\end{array}$ \\
\hline FCS Express & De Novo software & https://denovosoftware.com/ \\
\hline Matlab with Grafeo plugin & MathWorks & $\begin{array}{l}\text { https://www.mathworks.com/products/ } \\
\text { matlab.html, https://github.com/inatamara/ } \\
\text { Grafeo-dSTORM-analysis }\end{array}$ \\
\hline Chemdraw 16 & PerkinEImer & $\begin{array}{l}\text { https://www.perkinelmer.com/category/ } \\
\text { chemdraw }\end{array}$ \\
\hline Pro Fit & Quan Soft & https://www.quansoft.com \\
\hline ImageJ & Schneider et al., 2012 & https://imagej.nih.gov/ij/ \\
\hline
\end{tabular}

\section{RESOURCE AVAILABILITY}

\section{Lead contact}

Further information and requests for resources and reagents should be directed to and will be fulfilled by the Lead Contact, John Skidmore (js930@cam.ac.uk).

\section{Materials availability}

Plasmids for the production of RAD51 surrogate protein and BRC4 peptide are available from MH on request. Synthetic routes to all chemical compounds are described using established methodology from commercially available compounds. Where available these may be shared by the lead contact. Antibodies, reagents and cell lines used for the biological studies were obtained from the commercial or academic sources described in the attached Key resources table.

\section{Data and code availability}

All crystallographic statistics are shown in Table S3 and coordinates and structure factors deposited in the Protein Data Bank under accession numbers 6TV3, 6TWR, 6TW4 6XTW and 6TW9.

Software used for the biological studies were obtained from the commercial or academic sources described in the STAR methods Key resources table.

\section{EXPERIMENTAL MODEL AND SUBJECT DETAILS}

\section{E. coli for protein production}

For recombinant protein production the BL21(DE3) strain of E. coli (New England Biolabs, USA) was used in the Hyvönen Lab. Cells were grown in $2 \mathrm{YT}$ medium at $37^{\circ} \mathrm{C}$ until $\mathrm{OD}_{600}$ of $0.8-1.0$ after which the expression was induced with $400 \mu \mathrm{M}$ IPTG. Cells were grown for further 3 hours, centrifuged and stored frozen at $-20^{\circ} \mathrm{C}$. 
Cell line and cell culture

HCT116 colon carcinoma cells (male) and A549 lung adenocarcinoma cells (male) were obtained from ATCC and supplied mycoplasma free. HCT116 cells were maintained in McCoy's 5A (1x) + Glutamax-I growth medium (Gibco, 36600-021) supplemented with fetal bovine serum (FBS, Gibco Life Technologies, 10270-106) at a final concentration of 10\%. A549 cells were cultured in Dulbecco Modified Eagle medium (DMEM) (1x) +Glutamax-I (Gibco Life Technologies, 31966-021) with 10\% FBS. HeLa Kyoto cells (female, from the laboratory of Jonathan Pines, Institute of Cancer Research, London, U.K.), EUFA423 cells (male, from the European Fanconi Anemia Registry, VU University Medical Center, Amsterdam) and EUFA423+BRCA2 cells (Hattori et al., 2011) were cultured in DMEM with $10 \%$ FBS. All cells were grown at $37^{\circ} \mathrm{C} / 5 \% \mathrm{CO}_{2}$ in a humidified environment and all the assays were performed using these culturing conditions.

\section{METHOD DETAILS}

Chemical synthesis

See Methods S1 for synthetic methods for all compounds.

\section{Protein production}

All recombinant proteins used in this study (various HumRadA mutants and ChimRAD51) as well Alexa Fluor 488-labelled BRC repeat were prepared as described in detail in (Moschetti et al., 2016). The humanised RadA mutants, corresponding to residues 108-350 of Pyrococcus furiosus RadA, were expressed in BL21(DE3) strain of E. coli from T7-promoter driven plasmid pBAT4. Cells were grown in $2 Y T$ medium at $37^{\circ} \mathrm{C}$ until $\mathrm{OD}_{600}$ of $0.8-1.0$ after which the expression was induced with 400 uM IPTG. Cells were grown for further 3 hours, centrifuged and stored frozen at $-20^{\circ} \mathrm{C}$. Thawed cells were lysed in $20 \mathrm{mM} \mathrm{MES,} 0.5 \mathrm{mM}$ EDTA pH 6.0 buffer using Emulsiflex $\mathrm{C} 5$ homogeniser. The lysate was heated to $65^{\circ} \mathrm{C}$ for 10 minutes to denature most of the $E$. coli protein. The sample was centrifuged for 20-30 min at 15,000 xg and the soluble fraction from 1 liter of expression was loaded into a 5 ml HiTrap SP HP column (Cytiva) equilibrated with the lysis buffer. The bound proteins were eluted with a linear gradient to $0.5 \mathrm{M} \mathrm{NaCl}$ in $50 \mathrm{mM} \mathrm{MES} \mathrm{pH} \mathrm{6.0,} \mathrm{with} \mathrm{HumRadA}$ variants eluting at around $0.3 \mathrm{M}$ salt. The peak fractions were pooled and concentrated using centrifugal concentrators with $\mathrm{MWCO}$ of $10 \mathrm{kDa}$ to $2 \mathrm{ml}$. The sample was loaded into a Superdex 75 HiLoad 16/600 pg size exclusion column (Cytiva) which was equilibrated with $20 \mathrm{mM}$ MES, $100 \mathrm{mM} \mathrm{NaCl}, 0.5 \mathrm{mM}$ EDTA, pH 6.0. The samples eluted with an isocratic eluent at the expected position for their monomeric molecular weights as a single peak. The peak fractions were pooled, concentrated as before to typically $0.5 \mathrm{mM}$ concentration (as determined by UV absorbance at $280 \mathrm{~nm}$ using calculated molar absorption coefficient for each protein), flash frozen in liquid $\mathrm{N}_{2}$ in 25 ul aliquots and stored at $-80^{\circ} \mathrm{C}$.

The chimeric RAD51/RadA protein, ChimRAD51, used in FP assays was expressed as fusion construct containing a His-tag, GST, the BRC4 repeat in which the phenylalanine in the FxxA motif was mutated to alanine, a TEV cleavage site and ChimRAD51. The expression of this protein was carried out as describe above. After lysis of the cells and clarification of the lysate by centrifugation the soluble fraction was loaded into NiSepharose colun (Cytiva) under gravity and eluted after washing with $1000 \mathrm{mM}$ imidazole. The fusion protein was concentrated and purified further using a Superdex 75 HiLoad 16/600 pg size exclusion column (Cytiva) in $50 \mathrm{mM}$ Hepes/ $\mathrm{Na}$ (pH 8.0), $200 \mathrm{mM} \mathrm{KCl}, 100 \mathrm{mM}$ arginine, $100 \mathrm{mM}$ glutamate, and $100 \mathrm{mM}$ phenylalanine buffer. Peak fractions were flash frozen in liquid $\mathrm{N}_{2}$ and stored at $-80^{\circ} \mathrm{C}$ before TEV cleavage. After digestion with TEV the sample was re-purified by size exclusion chromatography using the same conditions, separating the monomeric ChimRAD51 from dimeric GST fusion part. This protein could be stored in the fridge for up to a week for FP assays. For ITC experiments the sample was passed through a NiSepharose column to capture any remaining His-GST fusion partner prior to use.

The labelled BRC4 repeat was synthesized using standard chemistry using Fmoc protection with an additional $N$-terminal cysteine which was used for labelling the peptide using maleimide Alexa Fluor 488 dye (ThermoFisher). The labelled protein was purified by reversed phase chromatography using a $4.6 \times 250 \mathrm{~mm}$ Ace C18 300 A column. The peptide was confirmed to be fully-labelled by



\section{ITC}

ITC was performed using a Microcal iTC200 instrument at $25^{\circ} \mathrm{C}$. Experiments typically involved titrating a 10-fold excess of ligand in the injection syringe against the protein ([HumRadA2] $=60 \mu \mathrm{M}$ or [ChimRAD51] $=20 \mu \mathrm{M}$ ) in either $200 \mathrm{mM}$ Tris buffer at pH 7.5 and $100 \mathrm{mM}$ $\mathrm{NaCl}$ (HumRadA2) or $20 \mathrm{mM}$ potassium phosphate at $\mathrm{pH} 8.0$ and $192 \mathrm{mM} \mathrm{KCl}$ (ChimRAD51). Titrations were typically performed with 5$10 \%$ DMSO and care was taken to ensure that the DMSO concentrations in the protein and ligand solutions were well matched. The raw ITC data were fitted using a single-site binding model in Microcal ITC LLC data analysis program in the Origin 7.0 package to derive the dissociation constant, stoichiometry and $\Delta \mathrm{H}$ of binding. For low affinity fragments stoichiometry was fixed to $1: 1$.

FP assay

Fluorescence Polarisation (FP) competition experiments were performed as described in (Moschetti et al., 2016). In brief, binding of $10 \mathrm{nM}$ Alexa Fluor 488-labelled BRC4 peptide to $50 \mathrm{nM}$ ChimRAD51 protein (giving approximately $80-90 \%$ saturation of binding) was competed with increasing concentration of inhibitor and the resulting competitive binding isotherms were measured and fitted using the expression described by (Wang, 1995) using Pro Fit software package (Quan Soft). 


\section{X-ray crystallography}

Crystallisation and structure determination was done as described in detail previously (Moschetti et al., 2016) using proteins produced in E. coli, as described above. Ligands were soaked into HumRadA1 or HumRadA22F crystals in the presence of cryo-protectant typically overnight and crystals cryo-cooled in liquid $\mathrm{N}_{2}$. Diffraction data was collected at Diamond and ESRF synchrotrons and processed with XDS or autoproc (Kabsch, 2010; Vonrhein et al., 2011). Structures were solved by molecular replacement using corresponding apo structures and ligands fitted into the emerging density after brief refinement and complex structures refined to completion using phenix.refine or autoBuster (Adams et al., 2010).

\section{Immunofluorescent visualisation of RAD51 foci/ $\gamma$ H2AX foci in A549 cells using the Cellomics Arrayscan V ${ }^{\text {ti }}$ high content microscopy}

A549 cells were seeded at 15000 cells/well in $100 \mu \mathrm{l}\left(1.5 \times 10^{5}\right.$ cells $\left./ \mathrm{ml}\right)$ in Nunc 96 -well plates (cat\# 167008) and grown overnight prior to the drug treatment. Compounds were added to cells such that the final DMSO concentration did not exceed $1 \% \mathrm{v} / \mathrm{v}$. Following compound addition, cells were exposed with specified levels of ionising radiation using the Xstrahl RS225 X-ray generator. After incubation with the compound for 6 hours, the medium was removed by aspiration and the cells washed twice in $1 \times$ PBS. Cells were fixed using fixative solution (4\% formaldehyde diluted in PBS) pre-warmed to $37^{\circ} \mathrm{C}$ for 10 min at room temperature. Cells were then washed twice in $100 \mu \mathrm{l} 1 \times \mathrm{PBS}$ at room temperature. Cells were then incubated in $100 \mu \mathrm{l}$ permeabilisation buffer for 5 minutes at room temperature after which they were incubated with $100 \mu$ l of blocking buffer ( $2 \%$ BSA (w/v), $0.2 \%$ Tween 20 (v/v), $0.1 \%$ Triton X-100 (v/ v) in PBS) for 90 minutes at room temperature. Cells were subsequently incubated with $50 \mu$ of mouse polyclonal anti-RAD51 Antibody (Abnova, cat \# H00005888-B01P) diluted 1:200 in blocking solution for $2 \mathrm{~h}$ at room temperature. Cells were washed in $100 \mu \mathrm{l}$ wash buffer at room temperature $(0.2 \%$ Tween $20(\mathrm{v} / \mathrm{v}), 0.1 \%$ Triton X-100 (v/v) in 1x PBS) then incubated in $50 \mu \mathrm{l}$ Alexa Fluor 488 labelled anti-mouse secondary antibody (1:500) and Hoechst 33342 (10 mg/ml stock) counterstain at 1:1000 in blocking solution for 60 minutes at room temperature. Finally, cells were washed twice in wash buffer and then twice in PBS and then stored in $100 \mu$ in PBS with a light protective seal at $4^{\circ} \mathrm{C}$ until read on the Cellomics Arrayscan $\mathrm{V}^{\mathrm{ti}}$ using a spot detector protocol. The number of cells analysed was 800 and the parameter used for analysis was Total Spot Area.

For detection of $\gamma \mathrm{H} 2 \mathrm{AX}$ foci in A549 cells, 10,000 cells/well $\left(1 \times 10^{5} \mathrm{cells} / \mathrm{ml}\right)$ were seeded in $100 \mu \mathrm{l}$ and left to grow overnight before treatment with compound. Cells were subsequently exposed to compounds and either $3 \mathrm{~Gy}$ ionising radiation or mock treated (left on the bench at room temperature). Staining protocol was identical as for RAD51 foci but anti-phospho $\gamma \mathrm{H} 2 \mathrm{AX}$ primary mouse monoclonal antibody was used (Millipore, cat\#05-636) at 1:2000 dilution. Image analysis was done using Cellomics software.

\section{SRB growth inhibition assay}

Adherent cell lines (HCT116 and A549 cells) were seeded into flat-bottomed tissue culture 96-well plates in a volume of $150 \mu \mathrm{L}$ of growth medium. HCT116 cells were seeded at 750 cells per well and A549 cells were seeded at 1000 cells per well. After 24 hours, compounds dissolved in DMSO were diluted in growth medium and were added to cells such that the final DMSO concentration was $1 \%(\mathrm{v} / \mathrm{v})$ and the final volume in the well was $200 \mu \mathrm{L}$. Cells were then incubated in the presence of compound for 96 hours before fixation.

Medium was removed from cells and $100 \mu \mathrm{L}$ of cold $1 \%(\mathrm{v} / \mathrm{v})$ trichloroacetic acid was added for 30 minutes at 4 degrees. The plates were washed three times in tap water and left to dry at room temperature. The fixed cells were stained in a $0.057 \%$ sulphorodamine B/ $1 \%$ acetic acid solution $(\mathrm{w} / \mathrm{v})$ and incubated at room temperature with agitation for 30 minutes after which the dye was removed and the plates washed in $1 \%(\mathrm{v} / \mathrm{v})$ acetic acid and left to dry. The dye was then solubilised in $10 \mathrm{mM}$ Tris solution (pH8) and incubated for 30 minutes under agitation. The plates were then read on a PHERAstar plus plate reader (BMG Labtech) using the fluorescence intensity module $\left(540-590 \mathrm{~nm}\right.$ ). Growth inhibition was calculated relative to DMSO controls and $\mathrm{Gl}_{50}$ values were calculated using Graphpad Prism.

For the PARP inhibitor experiments, the SRB method was used as described above to measure growth inhibition with the exception that cells were seeded into $150 \mu$ l medium and then a combination of either $25 \mu$ l of CAM833, AZD2461 (SelleckChem; \#S7029) or DMSO was added to give a total volume of $200 \mathrm{ul}$ in the well.

\section{Flow cytometry}

Propidium iodide staining solution (PI solution) was used at the following final concentrations: $200 \mathrm{ug} / \mathrm{ml}$ RNAase A (Sigma Aldrich, cat\# 10109169001), 0.1\% Triton-X 100 (v/v) and $20 \mathrm{ug} / \mathrm{ml}$ of propidium iodide solution diluted in $1 \times$ PBS. HCT116 cells were grown in 6 -well plates in a total volume of $2 \mathrm{ml}$ and treated with either test compound or DMSO control for the designated time. After treatment, medium was collected from the cells which were then washed in 1 XBS then removed from the plastic by the addition of in $500 \mu$ l Trypsin/EDTA until cells were monodispersed. The trypsin was neutralised by the removed media and the cell suspension was spun at $1000 \mathrm{rpm}$ for 5 minutes. Cells were then washed a further time in ice cold 1xPBS and spun at 1000 rpm for 5 minutes. Cells were then fixed in $4.5 \mathrm{ml} 70 \%$ ice cold ethanol and $0.5 \mathrm{ml}$ ice cold $1 \mathrm{xPBS}$. Cells were left in fixing solution overnight at $4{ }^{\circ} \mathrm{C}$ until processing. Cells were spun at $1000 \mathrm{rpm}$ for 5 mins and then washed in $1 \mathrm{xPBS}$, re-suspended in $0.5-1 \mathrm{ml}$ of the PI solution at incubated in the dark for 2 hours at room temperature. Cells were then counted and analysed using a Becton Dickinson LSR II cytometer and FCS Express software. 
Super-resolution microscopy

Single Molecule Localization Microscopy (SMLM) was achieved by direct Stochastic Optical Reconstruction Microscopy (d-STORM) as described (Haas et al., 2018). Briefly, samples were prepared for one colour 2D d-STORM utilizing a buffer containing 100 mM MEA-HCL (Sigma, M6500), 10\% glucose (Sigma), $0.5 \mathrm{mg} / \mathrm{ml}$ glucose oxidase (Sigma, G2133) and $40 \mu \mathrm{g} / \mathrm{ml}$ catalase (Sigma, $\mathrm{C100}$ ) in water at pH 7.5. Samples were imaged by direct STORM at room temperature in sealed 8-well ibidi $\mu$-slides utilizing an inverted N-STROM microscope (Nikon Ti, Japan) equipped with an Apochromat 100x/1.49 NA oil immersion objective. Samples were let to equilibrate for at least 30 minutes before imaging to minimize thermal drift. Images were then acquired with highly inclined illumination and focus was maintained by hardware autofocusing (Nikon Perfect Focus System). AlexaFluor647 was first pumped in its dark state using the $640 \mathrm{~nm}$ laser line at maximum power $(\sim 150 \mathrm{~mW})$ and then imaged continuously with a power density of $\sim 3 \mathrm{~kW} /$ $\mathrm{cm}^{2}$. Data were acquired in 'streaming mode' with a field-of-view (FOV) of 256x256 pixels (160 nm pixel size), at 65 frames per second for 25,000 frames with an EMCCD camera (iXon Ultra DU897, Andor). The sparsity of single molecules per frame was controlled using $\sim 0.6 \mathrm{~mW}$ of the $405 \mathrm{~nm}$ laser. Images of AlexaFluor647 were acquired with a Quad Band Set for TIRF applications (Chroma, TRF89901, ET - 405/488/561/640 nm) and the ET645/75 m emission filter (Chroma).

Cluster data analysis

Single molecule data was analysed using the Grafeo program available at https://github.com/inatamara/Grafeo-dSTORM-analysis-, as described in (Haas et al., 2018). Briefly, all localizations with fewer than 1,000 detected photons or localization precision lower than $20 \mathrm{~nm}$ were discarded. Next, the data was filtered using 2D Voronoi diagrams, setting the minimum density (an inverse of Voronoi polygon VP size) to $5^{\star} 10^{-5} \mathrm{~nm}^{-2}$. Finally, small isolated detections were supressed by thresholding univariate distance distribution function - a detection was rejected if it had less than 20 neighbours at the distance $\leq 100 \mathrm{~nm}$. Next, two-dimensional Delaunay triangulation (DT) was computed. Localizations were assigned to discreet clusters, connected components, by removing all DT edges larger than $20 \mathrm{~nm}$. All segmented connected components having less than 3 localizations were discarded. The number of RAD51 molecules inside a cluster was estimated by dividing the number of localization within a cluster by the expected number of localization obtained from isolated secondary antibodies used to label RAD51.

\section{mClover Lamin A HDR assay}

CAM833 was added to HeLa Kyoto cells grown on coverslips in 6 well plates 1 hour before transfection. Cells were transfected with sgRNA plasmid targeting Lamin A (pUC CBA-SpCas9.EF1a-BFP.sgLMNA, Addgene Plasmid \#98971) and donor plasmid (pCAGGS Donor mClover-LMNA, Addgene Plasmid \#98970) using JetPRIME transfection reagent (Polyplus Transfection). The next day, cell culture media were replaced with fresh media containing CAM833. Three days after transfection, cells were fixed with 4\% PFA for 10 minutes and permeabilized with TBS-Triton for 5 minutes before mounting. Images acquired with LSM 880 microscope were analyzed with Image $\mathrm{J}$ software (Schneider et al., 2012). HDR positive cells were defined as cells with mean mClover nuclear intensity over a threshold set for each experiment (Arnoult et al., 2017; Buisson et al., 2017).

\section{QUANTIFICATION AND STATISTICAL ANALYSES}

In the FP competition assay, each competitive binding isotherm resulted from averaging two replicate measurements from the same assay plate and this constituted a single independent measure of the binding isotherm. Per each candidate molecule we collected a minimum of three independently measured isotherms. These isotherms were averaged and then analysed by means of non-linear regression using the expression described by Wang (1995), implemented in Pro Fit software package (Quan Soft), that enabled us to estimate the pKd (the negative of the base-10 logarithm of the equilibrium dissociation constant) and the pKd's standard deviation. These values were used to initially rank compounds. Finally, each $\mathrm{pK}_{d}$ was converted into the corresponding equilibrium dissociation constant by exponentiation. For those candidate molecules that were measured repeatedly and were thus associated to a $n>3$, before exponentiation we used the $\mathrm{pK}_{d}$ estimates to calculate geometric average and Standard Deviations from the mean.

For microscopy experiments evaluating RAD51 and $\gamma-\mathrm{H} 2 \mathrm{AX}$ foci, analyses were with the Cellomics Arrayscan $\mathrm{V}^{\mathrm{ti}}$ instrument software, and for mClover Lamin A detection, with ImageJ software (Schneider et al., 2012). Figure 3 shows mean values \pm SEM of foci counts. Figure 5 shows the means, errors and tests of significance for mClover Lamin A detection. Details are provided in the Figure legend. For super-resolution microscopy cluster analysis in Figure 4B, simultaneous comparisons of the median values of multiple groups were performed using the Kruskal-Wallis test at the significance level alpha of 0.05 and familywise error rate was corrected by adjusting $\mathrm{p}$-values using the Tukey-Kramer method. Super-resolution image analysis was using the Grafeo program available at https://github.com/inatamara/Grafeo-dSTORM-analysis, as described in (Haas et al., 2018). Details are described in the Figure legend. For the SRB growth inhibition assay in Figures 5E, 6, and 7, Gl ${ }_{50}$ values were calculated using Graphpad Prism and are shown as data points and bars representing means and SEMs. Details are described in the Figure legends. 